TRANSACTIONS OF THE

AMERICAN MATHEMATICAL SOCIETY

Volume 284. Number 1. July 1984

\title{
MILNOR'S INVARIANTS AND THE COMPLETIONS OF LINK MODULES
}

BY

LORENZO TRALDI

\begin{abstract}
Let $L$ be a tame link of $\mu \geqslant 2$ components in $S^{3}, H$ the abelianization of its group $\pi_{1}\left(S^{3}-L\right)$, and $I H$ the augmentation ideal of the integral group $\operatorname{ring} \mathbf{Z} H$. The $I H$-adic completions of the Alexander module and Alexander invariant of $L$ are shown to possess presentation matrices whose entries are given in terms of certain integers $\mu\left(i_{1}, \ldots, i_{q}\right)$ introduced by J. Milnor. Various applications to the theory of the elementary ideals of these modules are given, including a condition on the Alexander polynomial necessary for the linking numbers of the components of $L$ with each other to all be zero. In the special case $\mu=2$, it is shown that the various Milnor invariants $\bar{\mu}([r+1, s+1])$ are determined (up to sign) by the Alexander polynomial of $L$, and that this Alexander polynomial is 0 iff $\bar{\mu}([r+1, s+1])=0$ for all $r, s \geqslant 0$ with $r+s$ even; also, the Chen groups of $L$ are determined (up to isomorphism) by those nonzero $\bar{\mu}([r+1, s+1])$ with $r+s$ minimal. In contrast, it is shown by example that for $\mu \geqslant 3$ the Alexander polynomials of a link and its sublinks do not determine its Chen groups.
\end{abstract}

1. Introduction. Let $L=K_{1} \cup \cdots \cup K_{\mu} \subseteq S^{3}$ be a tame link of $\mu \geqslant 2$ components; that is, $K_{1}, \ldots, K_{\mu}$ are pairwise disjoint, embedded copies of $S^{1}$, each of which is carried onto a polygonal curve by some autohomeomorphism of $S^{3}$. We presume that $L$ is given with a fixed ordering of its components (that is, each component $K_{i}$ has been assigned an index $i$ ), and also that each component of $L$ carries a fixed preferred orientation. Two such links are ambient isotopic iff there is an orientationpreserving autohomeomorphism of $S^{3}$ which maps one onto the other in such a way that the indices and orientations of the components correspond.

The group of such a link is the fundamental group $G=\pi_{1}\left(S^{3}-L\right)$ of its complement. The abelianization $H=G /[G, G]$ of $G$ is the free abelian group on certain elements $t_{1}, \ldots, t_{\mu}$, the meridians of $L$, and the elements of its integral group ring $\mathbf{Z} H$ may be uniquely represented by Laurent polynomials (with integer coefficients) in $t_{1}, \ldots, t_{\mu}$. The ring homomorphism $\varepsilon: \mathbf{Z} H \rightarrow \mathbf{Z}$ with $\varepsilon(h)=1 \quad \forall h \in H$ is the augmentation map; its kernel is the augmentation ideal $I H$ of $\mathbf{Z} H$.

The module sequence [5] of such a link $L$ is a short exact sequence

$$
0 \rightarrow B_{L} \stackrel{\phi}{\rightarrow} A_{L} \stackrel{\psi}{\rightarrow} I H \rightarrow 0
$$

Received by the editors January 25, 1983 and, in revised form, September 8, 1983 and December 10 , 1983. This paper was presented to the American Mathematical Society at its 806th meeting, in Fairfield, Connecticut, on October 28, 1983.

1980 Mathematics Subject Classification. Primary 57M05, 57M25.

Key words and phrases. Tame links, Milnor's invariants, $I$-adic completions, Chen groups, elementary ideals, Alexander polynomials. 
of $\mathbf{Z} H$-modules. The module $B_{L}$ is the abelianization $G^{\prime} / G^{\prime \prime}$ of the commutator subgroup $G^{\prime}=[G, G]$ of $G$, considered as an $H$-module via conjugation in $G$ (i.e., if $\alpha: G \rightarrow H$ is the natural epimorphism then for $\left.c \in G^{\prime}, \alpha(g) \cdot c G^{\prime \prime}=g c g^{-1} G^{\prime \prime}\right)$; following [18], we refer to $B_{L}$ as the Alexander invariant of $L$. The Alexander module of $L$ is the tensor product $A_{L}=\mathbf{Z} H \otimes_{\mathbf{Z} G} I G$, considered as a $\mathbf{Z} H$-module with scalar multiplication performed in the first factor. The maps $\phi$ and $\psi$ are given by $\phi\left(c G^{\prime \prime}\right)=1 \otimes(c-1)$ and $\psi(x \otimes y)=x \cdot \alpha(y)$. This sequence has been studied extensively by R. H. Crowell [4-6] and, in particular, may be identified [4] with the segment

$$
0 \rightarrow H_{1}(\tilde{X} ; \mathbf{Z}) \rightarrow H_{1}(\tilde{X}, F ; \mathbf{Z}) \rightarrow \tilde{H}_{0}(F ; \mathbf{Z}) \rightarrow 0
$$

of the reduced integral homology sequence of the pair $(\tilde{X}, F)$ consisting of the universal abelian cover $\tilde{X}$ of $S^{3}-L$ and its fiber $F$.

W. S. Massey [12] has studied the $I H$-adic completion

$$
0 \rightarrow \hat{B}_{L} \stackrel{\hat{\phi}}{\rightarrow} \hat{A}_{L} \stackrel{\hat{\psi}}{\rightarrow} \widehat{I H} \rightarrow 0
$$

of the module sequence of $L$, showing that it is a much less sensitive invariant of the link than the original module sequence. The completed sequence is invariant under (topological) $I$-equivalence [12, Theorem 4], for one thing. In addition, $\hat{B}_{L}$ and $\hat{A}_{L}$ are less complicated as $\widehat{\mathbf{Z H}}$-modules than $B_{L}$ and $A_{L}$ are as $\mathbf{Z} H$-modules, in that $\hat{B}_{L}$ and $\hat{A}_{L}$ have $\left(\left(\begin{array}{c}\mu \\ 3\end{array}\right)+\mu-1\right) \times\left(\begin{array}{c}\mu \\ 2\end{array}\right)$ and $(\mu-1) \times \mu$ presentation matrices respectively [12, Theorems 1 and 2], while there is no upper bound on the (finite) numbers of generators and reiations $B_{L}$ and $A_{L}$ may require [21, §4]. This completed module sequence is not so simple as to be uninteresting, though, as is evidenced by the fact [12] that for $q \geqslant 1$ the $(q+1)$ st Chen group of $L, G^{\prime \prime} G_{q+1} / G^{\prime \prime} G_{q+2}$, is isomorphic to $(\widehat{I H})^{q-1} \cdot \hat{B}_{L} /(\widehat{I H})^{q} \cdot \hat{B}_{L}$. (Here $G_{r}$ denotes the $r$ th lower central series subgroup of $G$, given by $G_{1}=G$ and $G_{r+1}=\left[G_{r}, G\right]$.) We will discuss these groups in $\S 7$.

The principal results of the present paper (Theorems (3.4) and (3.5)) give explicit presentation matrices for the $\overline{\mathrm{ZH}}$-modules $\hat{B}_{L}$ and $\hat{A}_{L}$; the entries of these matrices are elements of $\widehat{\mathbf{Z H}}$ defined using certain integers $\mu\left(i_{1}, \ldots, i_{q}\right)$ introduced by $\mathbf{J}$. Milnor [14] and discussed in $\S 2$. Let $\mathscr{M}$ be the $\mu \times \mu$ matrix with diagonal entries given by

$$
\mathscr{M}_{i i}=-\sum_{p=1}^{\infty} \sum \mu\left(i_{1}, \ldots, i_{p}, i\right) \cdot \prod\left(t_{i_{k}}-1\right)
$$

the sum $\sum$ taken over the set of those $p$-tuples $\left(i_{1}, \ldots, i_{p}\right)$ of elements of $\{1, \ldots, \mu\}$ with $i_{p} \neq i$, and other entries given by

$$
\mathscr{M}_{i j}=\mu(j, i) \cdot\left(t_{i}-1\right)+\sum_{p=1}^{\infty} \sum \mu\left(i_{1}, \ldots, i_{p}, j, i\right) \cdot\left(t_{i}-1\right) \cdot \prod\left(t_{i_{h}}-1\right),
$$


the sum $\sum$ now taken over the set of all $p$-tuples $\left(i_{1}, \ldots, i_{p}\right)$ of elements of $\{1, \ldots, \mu\}$. Theorem (3.4) then states that $\mathscr{M}$ is a presentation matrix for the $\widehat{\mathbf{Z H}}$-module $\hat{A}_{L}$, and also that if any single row of $\mathscr{M}$ is deleted the resulting matrix is still a presentation matrix for $\hat{A}_{L}$. Theorem (3.5), which we need not repeat here, gives a related presentation matrix $\mathscr{P}$ for $\hat{B}_{L}$.

Theorem (3.4) has several interesting applications to the theory of the elementary ideals of $A_{L}$. The most direct, Theorem (4.1), states that if we regard $\mathbf{Z} H$ as a

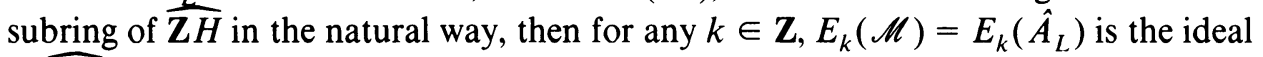
of $\widehat{\mathbf{Z H}}$ generated by $E_{k}\left(A_{L}\right)$. It is also possible to obtain results within the ring $\mathbf{Z} H$, rather than the larger ring $\widehat{\mathbf{Z H}}$. For instance, if for $q \geqslant 1$ we let $\mathscr{M}_{q}$ be the matrix obtained by restricting the index of summation $p$ in the definition of $\mathscr{M}_{i i}$ to values $<q$, and restricting $p$ in the definition of $\mathscr{M}_{i j}$ to values $<q-1$ (so that, for example, $\mathscr{M}_{1}=0$ ), then Theorem (4.2) states that for $0<k<\mu$

$$
\sum_{i=0}^{k-1} E_{\mu-k+i}\left(A_{L}\right) \cdot(I H)^{q i}+(I H)^{q k}=\sum_{i=0}^{k-1} E_{\mu-k+i}\left(\mathscr{M}_{q}\right) \cdot(I H)^{q i}+(I H)^{q k} .
$$

A simple consequence of this is Corollary (4.3):

$$
E_{\mu-k}\left(A_{L}\right)+(I H)^{k+q-1}=E_{\mu-k}\left(\mathscr{M}_{q}\right)+(I H)^{k+q-1} .
$$

Analogous results hold for the elementary ideals of $B_{L}$ (see $\S 4$ ).

M. E. Kidwell [11] has given a condition on the reduced Alexander polynomial that is necessary for the linking numbers $\mu(i, j)$ to be zero for all $i \neq j \in\{1, \ldots, \mu\}$. Using Theorem (4.2), we lift Kidwell's condition to the (unreduced) Alexander polynomial $\Delta_{1}\left(A_{L}\right)=\Delta_{0}\left(B_{L}\right)$ in Theorem (5.3): if $\mu(i, j)=0 \forall i \neq j \in\{1, \ldots, \mu\}$, then either $\mu$ is even and $\Delta_{1}\left(A_{L}\right) \in(I H)^{2 \mu-2}$, or $\mu$ is odd and there is an $x \in(I H)^{(\mu-3) / 2}$ with

$$
\left(\Delta_{1}\left(A_{L}\right)\right)+(I H)^{2 \mu-2}=\left(x^{2} \cdot \prod_{i=\alpha_{i}}^{\mu}\left(t_{i}-1\right)\right)+(I H)^{2 \mu-2} .
$$

(Here for $y \in \mathbf{Z} H,(y)$ denotes the principal ideal of $\mathbf{Z} H$ generated by $y$.)

In $\$ 6$ we concentrate, for the most part, on the case $\mu=2$. In this case Theorem (4.1) amounts to the statement that the Alexander polynomial $\Delta_{1}\left(A_{L}\right)$ generates the same principal ideal in $\widehat{\mathbf{Z H}}$ as either

$$
\mathscr{M}_{12} /\left(t_{1}-1\right)=\mathscr{M}_{11} /\left(1-t_{2}\right)
$$

or

$$
\mathscr{M}_{21} /\left(t_{2}-1\right)=\mathscr{M}_{22} /\left(1-t_{1}\right) \text {. }
$$

From this and certain well-known properties of the integers $\mu\left(i_{1}, \ldots, i_{q}\right)$ we deduce Theorem (6.4): if for $u, v \geqslant 1$ we let $[u, v]=(1, \ldots, 1,2, \ldots, 2)$ be the sequence with $u$ ones and $v$ twos, then after multiplying $\Delta_{1}\left(A_{L}\right)$ by -1 (if necessary) it will be true that for all $r, s \geqslant 0$

$$
\frac{1}{r ! s !} \varepsilon\left(\frac{d^{r+s}}{d t_{1}^{r} d t_{2}^{s}}\left(\Delta_{1}\left(A_{L}\right)\right)\right) \equiv(-1)^{s} \mu([r+1, s+1]) \quad(\operatorname{modulo} \Delta([r+1, s+1])) \text {. }
$$


Here $d / d t_{1}$ and $d / d t_{2}$ are the usual partial derivatives of multivariate calculus, and $\Delta([r+1, s+1]) \in \mathbf{Z}$ is the g.c.d. of those $\mu([u, v])$ with $u \leqslant r+1, v \leqslant s+1$ and $u+v \leqslant r+s+1$. (This result is a strengthening of a theorem due to K. Murasugi [15, Theorem 4.1], and is itself due to N. Smythe [19].) An immediate consequence is Corollary (6.5): for $t \geqslant 0, \Delta_{1}\left(A_{L}\right) \in(I H)^{t}$ iff $\mu([r+1, s+1])=0$ whenever $r+s$ $<t$, and (hence) $\Delta_{1}\left(A_{L}\right)=0$ iff $\mu([r+1, s+1])=0 \forall r, s \geqslant 0$. (In fact, for $\Delta_{1}\left(A_{L}\right)$ to be 0 it suffices that $\mu([r+1, s+1])$ be 0 for all those $r, s \geqslant 0$ with $r+s$ even; see §6.) We also generalize the property (mentioned in passing by J. H. Conway [3]) that a + amphicheiral two-component link has Alexander polynomial 0.

Finally, in $\S 8$ we discuss questions suggested by the results of the paper.

We would like to express our gratitude to the Committee on Advanced Study and Research of Lafayette College, under whose auspices this investigation was carried out. Further thanks are due to J. W. Cogdell, J. A. Hillman and especially the referee, for their encouragement and suggestions.

2. The integers $\mu\left(i_{1}, \ldots, i_{q}\right)$. As is well known, if $L=K_{1} \cup \cdots \cup K_{\mu} \subseteq S^{3}$ is a tame link, a presentation of the group $G=\pi_{1}\left(S^{3}-L\right)$ may be obtained from any regular projection of $L$ in the plane. The arc components of such a projection may be denoted $e_{i j}\left(1 \leqslant i \leqslant \mu, 1 \leqslant j \leqslant j_{i}\right)$, in such a way that for each $i, e_{i 1} \cup \cdots \cup e_{i j_{i}}$ is the image of $K_{i}$ in the projection, and $e_{i 1}, \ldots, e_{i j_{i}}$ are encountered successively as one traverses $K_{i}$ in the preferred direction. (The index $j$ of $e_{i j}$ should be considered modulo $j_{i}$.) $G$ then has the presentation $\left\langle x_{i j} ; r_{i j}\right\rangle$, in which there is a generator $x_{i j}$ whenever $1 \leqslant i \leqslant \mu$ and $1 \leqslant j \leqslant j_{i}$, and a relator $r_{i j}=x_{m n}^{\delta_{i j}} x_{i j} x_{m n}^{-\delta_{i j}} x_{i j+1}^{-1}$ whenever $e_{m n}$ is the arc component separating the terminal endpoint of $e_{i j}$ from the initial endpoint of $e_{i j+1}$; here $\delta_{i j}$ is 1 or -1 , depending on whether $e_{m n}$ is oriented from left to right or from right to left, as seen by an observer standing on $e_{i j}$ and facing $e_{i j+1}$. Any one of these relators is redundant [18, §3.D], and can be deleted from the presentation without effect.

If $F$ is the free group on $\left\{x_{i j} \mid 1 \leqslant i \leqslant \mu, 1 \leqslant j \leqslant j_{i}\right\}$, then there is an epimorphism $\eta: F \rightarrow G$ whose kernel is the normal subgroup of $F$ generated by $\left\{r_{i j}\right\}$. If $\alpha$ : $G \rightarrow H$ is the natural epimorphism, then clearly $\alpha \eta\left(x_{i j}\right)=\alpha \eta\left(x_{i k}\right)$ whenever $1 \leqslant i$ $\leqslant \mu$ and $1 \leqslant j, k \leqslant j_{i}$, and the elements $t_{i}=\alpha \eta\left(x_{i 1}\right), 1 \leqslant i \leqslant \mu$, constitute a basis of the free abelian group $H$.

If $q \geqslant 1$, it is a simple matter to obtain a presentation $\left\langle x_{i j} ; r_{i j}, s_{k q+1}\right\rangle$ of $G / G_{q+1}$, by adjoining sufficiently many relators $s_{k q+1}$ to generate the normal subgroup $F_{q+1}$ of $F$. A less cumbersome presentation for $G / G_{q+1}$ has been found by J. Milnor [14]. If $\Phi \subseteq F$ is the subgroup (freely) generated by $\left\{x_{i}=x_{i 1} \mid 1 \leqslant i \leqslant \mu\right\}$, and $\beta_{q+1}: G \rightarrow G / G_{q+1}$ is the natural epimorphism, he shows that the restriction $\beta_{q+1} \eta \mid \Phi: \Phi \rightarrow G / G_{q+1}$ is surjective, and its kernel is generated (as a normal subgroup of $\Phi$ ) by $\Phi_{q+1}$ together with certain elements $\rho_{i q+1}=\left[x_{i}, w_{i q+1}\right], 1 \leqslant i \leqslant \mu$. Thus $G / G_{q+1}$ has the presentation $\left\langle x_{i} ; \rho_{i q+1}, \sigma_{k q+1}\right\rangle$, in which there are a generator $x_{i}$ and a relator $\rho_{i q+1}$ for $1 \leqslant i \leqslant \mu$, and sufficiently many relators $\sigma_{k q+1}$ to generate the normal subgroup $\Phi_{q+1}$ of $\Phi$. The element $w_{i q+1} \in \Phi$ depends on the integer $q$; if $p \geqslant q$ then $w_{i p+1}$ can be substituted for $w_{i q+1}$, since the two are congruent modulo 
$\Phi_{q}\left[14, \S 3\right.$, assertion (6)], and hence $\left[x_{i}, w_{i q+1}\right]$ and $\left[x_{i}, w_{i p+1}\right]$ are congruent modulo $\Phi_{q+1}$. (Incidentally, we might note here that it is not difficult to slightly sharpen the arguments of $[14, \S 3$, assertions (4) and (6)], so that (in the notation of [14]) one can take $w_{i q+1}=\eta_{q-1}\left(v_{i r_{i}}\right)$ rather than $\eta_{q+1}\left(v_{i r_{i}}\right)$, where $\eta_{0} \equiv 1$.) Also, since the presentation $\left\langle x_{i} ; \rho_{i q+1}, \sigma_{k q+1}\right\rangle$ of $G / G_{q+1}$ is derived from the presentation $\left\langle x_{i j} ; r_{i j}\right\rangle$ of $G$, the redundancy property of the latter implies that any one relator $\rho_{i q+1}$ in the former is redundant.

If $q \geqslant 2$, then for each $q$-tuple $\left(i_{1}, \ldots, i_{q}\right)$ of elements of $\{1, \ldots, \mu\}$ an integer $\mu\left(i_{1}, \ldots, i_{q}\right)$ is defined in terms of R. H. Fox's free derivatives [7] by the formula

$$
\mu\left(i_{1}, \ldots, i_{q}\right)=\varepsilon\left(\frac{\partial^{q-1}}{\partial x_{i_{1}} \cdots \partial x_{i_{q-1}}}\left(w_{i_{q} q+1}\right)\right),
$$

where $\varepsilon: \mathbf{Z} F \rightarrow \mathbf{Z}$ is the augmentation map. In the simplest case, $q=2, \mu\left(i_{1}, i_{2}\right)$ is either zero (if $i_{1}=i_{2}$ ) or the linking number of $K_{i_{1}}$ and $K_{i_{2}}$ (if $i_{1} \neq i_{2}$ ). For $q \geqslant 3$, $\mu\left(i_{1}, \ldots, i_{q}\right)$ is not, in general, an invariant of the link $L$; rather, it depends on the choice of a regular projection of $L$ in the plane, and the choice of an indexing of the arc components of the projection. J. Milnor has shown, however, that if $\Delta\left(i_{1}, \ldots, i_{q}\right)$ is the greatest common divisor of the integers $\mu\left(j_{1}, \ldots, j_{p}\right)$, as $\left(j_{1}, \ldots, j_{p}\right)$ varies over the proper subsequences of $\left(i_{1}, \ldots, i_{q}\right)$, then $\Delta\left(i_{1}, \ldots, i_{q}\right)$ and the congruence class $\bar{\mu}\left(i_{1}, \ldots, i_{q}\right)$ of $\mu\left(i_{1}, \ldots, i_{q}\right)$ modulo $\Delta\left(i_{1}, \ldots, i_{q}\right)$ are not only invariants of $L$, but isotopy invariants of $L$ [14]. (This definition of $\Delta\left(i_{1}, \ldots, i_{q}\right)$ differs from that given in [14]; that the two agree is a consequence of the "cyclic symmetry" of Milnor's invariants [14, Theorem 6].) Furthermore, if $i_{1}, \ldots, i_{q}$ are pairwise distinct then $\bar{\mu}\left(i_{1}, \ldots, i_{q}\right)$ is a homotopy invariant of $L[14$, Theorem 8]. A weaker version of this invariant $\bar{\mu}\left(i_{1}, \ldots, i_{q}\right)$ may be obtained by considering its congruence class $\mu^{*}\left(i_{1}, \ldots, i_{q}\right)$ modulo $\Delta^{*}\left(i_{1}, \ldots, i_{q}\right)$, the greatest common divisor of the integers $\mu\left(j_{1}, \ldots, j_{p}\right)$ as $\left(j_{1}, \ldots, j_{p}\right)$ varies over the permutations of proper subsequences of $\left(i_{1}, \ldots, i_{q}\right)$.

3. Presentations of $\hat{A}_{L}$ and $\hat{B}_{L}$. The Alexander module of the group $G$ is defined to be the tensor product $\mathbf{Z} H \otimes_{\mathbf{Z} G} I G$, considered as a $\mathbf{Z} H$-module with the scalar multiplication performed in the first factor; we denote this module $A_{L}$. For $q \geqslant 1$ the Alexander module of $G / G_{q+1}$ is defined analogously; we identify $H$ with the abelianization of $G / G_{q+1}$ in the natural way and consider this Alexander module as a $\mathbf{Z} H$-module, which we will denote $A_{q+1}$.

The Alexander matrix of the presentation $\left\langle x_{i j} ; r_{i j}\right\rangle$ of $G$ has a column for each generator $x_{i j}$, and a row for each relator $r_{i j}$; the common entry of the column corresponding to $x_{m n}$ and the row corresponding to $r_{i j}$ is

$$
\alpha \eta\left(\partial r_{i j} / \partial x_{m n}\right) \text {. }
$$

As shown by R. H. Crowell $[4, \S 3]$, this matrix is a presentation matrix for the $\mathbf{Z} H$-module $A_{L}$. In fact, if $X_{2}$ is the free $\mathbf{Z} H$-module on the set of relators $r_{i j}$, and $X_{1}$ is the free $\mathbf{Z} H$-module on the set of generators $x_{i j}$, then there is an exact sequence

$$
X_{2} \stackrel{d_{2}}{\rightarrow} X_{1} \stackrel{d_{1}}{\rightarrow} A_{L} \rightarrow 0
$$


in which the matrix of $d_{2}$ is this Alexander matrix, and $d_{1}\left(x_{i j}\right)=1 \otimes\left(\eta\left(x_{i j}\right)-1\right)$. Similarly, if $q \geqslant 1$ each of the presentations $\left\langle x_{i j} ; r_{i j}, s_{k q+1}\right\rangle$ and $\left\langle x_{i} ; \rho_{i q+1}, \sigma_{k q+1}\right\rangle$ of the group $G / G_{q+1}$ gives rise to a presentation of the $\mathbf{Z} H$-module $A_{q+1}$.

LEMMA (3.1). Let $\partial: F \rightarrow \mathbf{Z} F$ be a derivation, that is, a function with $\partial(x y)=\partial(x)$ $+x \partial(y) \forall x, y \in F$. Then $\alpha \eta \partial\left(F_{q+1}\right) \subseteq(I H)^{q}$ for every $q \geqslant 1$.

Proof. Note that $\partial(1)=\partial(1)+\partial(1)$, so $\partial(1)=0 ;$ hence $\partial\left(x^{-1}\right)=-x^{-1} \partial(x)$ $\forall x \in F$. It follows that for $x, y \in F$

$$
\alpha \eta \partial([x, y])=\alpha \eta \partial\left(x y x^{-1} y^{-1}\right)=(1-\alpha \eta(y)) \alpha \eta \partial(x)+(\alpha \eta(x)-1) \alpha \eta \partial(y) \text {. }
$$

From this we conclude directly that $\alpha \eta \partial\left(F_{2}\right) \subseteq I H$.

Proceeding inductively, suppose $q \geqslant 2$. If $x \in F_{q}$ and $y \in F$, then

$$
\alpha \eta \partial([x, y])=(1-\alpha \eta(y)) \alpha \eta \partial(x) \in(I H)^{q} \text {. Q.E.D. }
$$

We will apply this lemma with the various free derivatives $\partial / \partial x_{i j}$ playing the role of $\partial$.

For $q \geqslant 1$, let $\beta_{q+1}: G \rightarrow G / G_{q+1}$ be the canonical map onto the quotient; we also denote by $\beta_{q+1}$ the map induced on the integral group rings, and the restriction of this map to the augmentation ideals.

Proposition (3.2). For $q \geqslant 1$, id $\otimes \beta_{q+1}: A_{L} \rightarrow A_{q+1}$ induces an isomorphism

$$
A_{L} /(I H)^{q} \cdot A_{L} \rightarrow A_{q+1} /(I H)^{q} \cdot A_{q+1} \text {. }
$$

Proof. Let

$$
X_{2}^{\prime} \stackrel{d_{2}^{\prime}}{\rightarrow} X_{1}^{\prime} \stackrel{d_{1}^{\prime}}{\rightarrow} A_{q+1} \rightarrow 0
$$

be the presentation of the $\mathbf{Z} H$-module $A_{q+1}$ arising from the presentation $\left\langle x_{i j} ; r_{i j}, s_{k q+1}\right\rangle$ of $G / G_{q+1}$. Comparing this with the presentation of $A_{L}$ discussed earlier, we note that $X_{1}=X_{1}^{\prime}$ and (id $\left.\otimes \beta_{q+1}\right) d_{1}=d_{1}^{\prime}$. Furthermore, by the lemma,

$$
d_{2}\left(X_{2}\right) \subseteq d_{2}^{\prime}\left(X_{2}^{\prime}\right) \subseteq d_{2}\left(X_{2}\right)+(I H)^{q} \cdot X_{1} \text {. Q.E.D. }
$$

Suppose $q \geqslant 1$, and recall the matrix $\mathscr{M}_{q}$ that was defined in the introduction. Let $Y$ be the free $\mathbf{Z} H$-module on the set $\left\{x_{1}, \ldots, x_{\mu}\right\}$, and for $1 \leqslant r \leqslant \mu$ let $Y_{r}$ be the submodule of $Y$ (freely) generated by those $x_{i} \neq x_{r}$; let $Y_{0}=Y$. For $1 \leqslant r \leqslant \mu$ let $e_{r q}: Y_{r} \rightarrow Y$ be the $\mathbf{Z} H$-linear map whose matrix is the submatrix of $\mathscr{M}_{q}$ obtained by deleting its $r$ th row, and let $e_{0 q}: Y_{0} \rightarrow Y$ be the $\mathrm{Z} H$-linear map whose matrix is $\mathscr{M}_{q}$; also, let $e_{q}: Y \rightarrow A_{q+1}$ be the homomorphism given by $e_{q}\left(x_{i}\right)=1 \otimes\left(\beta_{q+1} \eta\left(x_{i}\right)-1\right)$. We use $\bar{e}$ to denote the homomorphism induced by a homomorphism $e$ from some quotient of its domain to a suitable quotient of its codomain.

Proposition (3.3). For every $q \geqslant 1$ and every $r \in\{0, \ldots, \mu\}$ the sequence

$$
Y_{r} /(I H)^{q} \cdot Y_{r} \stackrel{\bar{e}_{r q}}{\rightarrow} Y /(I H)^{q} \cdot Y \stackrel{\bar{e}_{q}}{\rightarrow} A_{q+1} /(I H)^{q} \cdot A_{q+1} \rightarrow 0
$$

is exact.

Proof. Suppose, first, that $r=0$, and let

$$
X \stackrel{f_{q}}{\rightarrow} Y \stackrel{e_{q}}{\rightarrow} A_{q+1} \rightarrow 0
$$


be the presentation of the $\mathbf{Z H}$-module $A_{q+1}$ that arises from the presentation $\left\langle x_{i} ; \rho_{i q+1}, \sigma_{k q+1}\right\rangle$ of $G / G_{q+1}$. Then $X$ is the free $\mathbf{Z} H$-module on the set of relators $\rho_{i q+1}$ and $\sigma_{k q+1}$, and, by Lemma (3.1), $f_{q}\left(\sigma_{k q+1}\right) \in(I H)^{q} \cdot Y$ for each $k$. Let $X_{0}$ be the submodule of $X$ (freely) generated by the set of relators $\rho_{i q+1}$, and $g_{q}: X_{0} \rightarrow Y$ the restriction of $f_{q}$ to $X_{0}$; then

$$
X_{0} /(I H)^{q} \cdot X_{0} \stackrel{\bar{g}_{q}}{\rightarrow} Y /(I H)^{q} \cdot Y \stackrel{\bar{e}_{q}}{\rightarrow} A_{q+1} /(I H)^{q} \cdot A_{q+1} \rightarrow 0
$$

is an exact sequence of $\mathbf{Z} H$-modules.

To complete the proof in this case $r=0$, then, we need only show that $\bar{g}_{q}$ and $\bar{e}_{0 q}$ have the same image. If $q=1, \bar{g}_{q}=0$ and $\bar{e}_{0 q}=0$, so we need proceed no further. It happens that for $q \geqslant 2$ and $1 \leqslant i, j \leqslant \mu$ the $i j$ entry of $\mathscr{M}_{q}$ is congruent, modulo $(I H)^{q}$, to the $i j$ entry of the matrix of $g_{q}$. To show this we need to recall R. H. Fox's "Taylor series" [7, p. 553], which asserts that for every integer $s \geqslant 1$ and every $x \in \mathbf{Z} \Phi$

$$
x-\varepsilon(x)-\sum_{p=1}^{s} \sum \varepsilon\left(\partial^{p}(x) / \partial x_{i_{1}} \cdots \partial x_{i_{p}}\right) \cdot \Pi\left(x_{i_{k}}-1\right)
$$

is an element of $(I \Phi)^{s+1}$, where the sum $\sum$ is taken over the set of all $p$-tuples $\left(i_{1}, \ldots, i_{p}\right)$ of elements of $\{1, \ldots, \mu\}$.

If $1 \leqslant i, j \leqslant \mu$ the $i j$ entry of the matrix of $g_{q}$ is

$$
\begin{aligned}
\alpha \eta\left(\partial\left(\rho_{i q+1}\right) / \partial x_{j}\right)= & \left(1-\alpha \eta\left(w_{i q+1}\right)\right) \alpha \eta\left(\partial\left(x_{i}\right) / \partial x_{j}\right) \\
& +\left(\alpha \eta\left(x_{i}\right)-1\right) \alpha \eta\left(\partial\left(w_{i q+1}\right) / \partial x_{j}\right) .
\end{aligned}
$$

Applying the "Taylor series" to $w_{i q+1}$ (with $\left.s=q-1\right)$ and $\partial\left(w_{i q+1}\right) / \partial x_{j}$ (with $s=q-2$ ), we conclude that the $i j$ entry of the matrix of $g_{q}$ is congruent (modulo $\left.(I H)^{q}\right)$ to

$$
\begin{aligned}
& -\alpha \eta\left(\partial\left(x_{i}\right) / \partial x_{j}\right) \cdot \sum_{p=1}^{q-1} \sum \mu\left(i_{1}, \ldots, i_{p}, i\right) \cdot \prod\left(t_{i_{k}}-1\right) \\
& \quad+\left(t_{i}-1\right) \cdot \mu(j, i)+\sum_{p=1}^{q-2} \sum \mu\left(i_{1}, \ldots, i_{p}, j, i\right) \cdot\left(t_{i}-1\right) \cdot \prod\left(t_{i_{k}}-1\right),
\end{aligned}
$$

where each sum $\sum$ is taken over the set of all $p$-tuples $\left(i_{1}, \ldots, i_{p}\right)$ of elements of $\{1, \ldots, \mu\}$. Since this element of $\mathbf{Z} H$ is precisely the $i j$ entry of $\mathscr{M}_{q}$, our proof in the case $r=0$ is complete.

Suppose, now, that $1 \leqslant r \leqslant \mu$. As noted in $\S 2$, in the presentation $\left\langle x_{i} ; \rho_{i q+1}, \sigma_{k q+1}\right\rangle$ of $G / G_{q+1}$ the relator $\rho_{r q+1}$ is redundant, and so may be deleted. Applying the argument just given in the case $r=0$ to this simplified presentation completes the proof. Q.E.D.

We are indebted to the referee for pointing out that the exact sequence of Proposition (3.3) has been described by N. Smythe [19].

Recall that for a $\mathbf{Z} H$-module $A$ the $I H$-adic completion $\hat{A}$ may be identified with the inverse limit of the quotients $A /(I H)^{q} \cdot A[1$, III, \$2.6]; the canonical epimorphisms $A \rightarrow A /(I H)^{q} \cdot A$ then define a natural mapping $h: A \rightarrow \hat{A}$, whose kernel is 
$\bigcap_{q=0}^{\infty}(I H)^{q} \cdot A$. In case $h$ is injective (e.g., if $A$ is free) we may suppress it, regarding $A$ as a subset of $\hat{A}$. If $A$ is finitely generated, there is an isomorphism $\widehat{\mathbf{Z H}} \otimes_{\mathrm{Z} H} A \rightarrow \hat{A}$ under which $x \otimes a$ corresponds to $x \cdot h(a)$ [1, III, §3.4]. From the additivity of tensor products it follows that if $A$ is the free $\mathbf{Z} H$-module on a finite set $\left\{a_{1}, \ldots, a_{n}\right\}$, $\hat{A}$ is the free $\widehat{\mathbf{Z H}}$-module on the same set.

Continuing to use the notation of Proposition (3.3), let $f: \hat{Y} \rightarrow \hat{A}_{L}$ be the $\widehat{\mathbf{Z H}}$-homomorphism with $f\left(x_{i}\right)=h\left(1 \otimes\left(\eta\left(x_{i}\right)-1\right)\right)$. Let $e_{0}: \hat{Y}_{0} \rightarrow \hat{Y}$ be the $\widehat{\mathbf{Z H}}$-homomorphism whose matrix is $\mathscr{M}$, and for $1 \leqslant r \leqslant \mu$ let $e_{r}: \hat{Y}_{r} \rightarrow \hat{Y}$ be the homomorphism whose matrix is obtained from $\mathscr{M}$ by deleting its $r$ th row.

THEOREM (3.4). For every $r \in\{0, \ldots, \mu\}$ the sequence

$$
\hat{Y}_{r} \stackrel{e_{r}}{\rightarrow} \hat{Y} \stackrel{f}{\rightarrow} \hat{A}_{L} \rightarrow 0
$$

is exact.

Proof. Let $r \in\{0, \ldots, \mu\}$. Combining Propositions (3.2) and (3.3), we see that for every $q \geqslant 1$ the sequence

$$
Y_{r} /(I H)^{q} \cdot Y_{r} \stackrel{\bar{e}_{r q}}{\rightarrow} Y /(I H)^{q} \cdot Y \stackrel{\bar{f}_{q}}{\rightarrow} A_{L} /(I H)^{q} \cdot A_{L} \rightarrow 0
$$

is exact, where $f_{q}: Y \rightarrow A_{L}$ is the $\mathbf{Z} H$-homomorphism given by $f_{q}\left(x_{i}\right)=1 \otimes\left(\eta\left(x_{i}\right)\right.$ -1 ). (The sequence of the statement is the inverse limit of these sequences as $q \rightarrow \infty$.)

Let $\pi: \hat{Y} \rightarrow$ coker $e_{r}$ be the canonical map onto the quotient. Certainly $f e_{r}=0$, so there is a $\widehat{\mathbf{Z H}}$-homomorphism $\hat{f}$ : coker $e_{r} \rightarrow \hat{A}_{L}$ with $\hat{f} \pi=f$. Recall that for any finitely generated $\mathbf{Z} H$-module $A$ the natural mapping $h: A \rightarrow \hat{A}$ induces an isomorphism $A /(I H)^{q} \cdot A \rightarrow \hat{A} /(\widehat{I H})^{q} \cdot \hat{A}$ for every $q \geqslant 1[1$, III, §2.12]. From this and the exact sequences of the first paragraph, we deduce that $\hat{f}$ induces an isomorphism

$$
\text { coker } e_{r} /(\widehat{I H})^{q} \cdot \text { coker } e_{r} \rightarrow \hat{A}_{L} /(\widehat{I H})^{q} \cdot \hat{A}_{L}
$$

for every $\mathrm{q} \geqslant 1$; it follows that $\hat{f}$ is an isomorphism [1, III, §2.8, Corollary 3]. Q.E.D.

A presentation of the $\widehat{\mathbf{Z H}}$-module $\hat{B}_{L}$ may be derived from that of $\hat{A}_{L}$ just given, using a construction due to R. H. Crowell $[5,6]$. Let $Z_{1}, Z_{2}, Z_{3}$ be the free $\mathbf{Z} H$-modules on the sets $\left\{z_{i} \mid 1 \leqslant i \leqslant \mu\right\},\left\{z_{i j} \mid 1 \leqslant i<j \leqslant \mu\right\}$, and $\left\{z_{i j k} \mid 1 \leqslant i<j\right.$ $<k \leqslant \mu\}$, respectively (if $\mu=2, Z_{3}=0$ ). There is an exact sequence

$$
Z_{3} \stackrel{\zeta_{3}}{\rightarrow} Z_{2} \stackrel{\zeta_{2}}{\rightarrow} Z_{1} \stackrel{\zeta_{1}}{\rightarrow} I H \rightarrow 0
$$

in which $\zeta_{1}\left(z_{i}\right)=t_{i}-1, \zeta_{2}\left(z_{i j}\right)=\left(t_{i}-1\right) z_{j}-\left(t_{j}-1\right) z_{i}$, and $\zeta_{3}\left(z_{i j k}\right)=\left(t_{i}-1\right) z_{j k}$ $-\left(t_{j}-1\right) z_{i k}+\left(t_{k}-1\right) z_{i j}$. We denote the matrix of $\zeta_{2} N_{2}(\mu)$, and that of $\zeta_{3} N_{3}(\mu)$ (in particular, $N_{3}(2)$ is the empty matrix). The $I H$-adic completion

$$
\hat{Z}_{3} \stackrel{\hat{\zeta}_{3}}{\rightarrow} \hat{Z}_{2} \stackrel{\hat{\zeta}_{2}}{\rightarrow} \hat{Z}_{1} \stackrel{\hat{\zeta}_{1}}{\rightarrow} \widehat{I H} \rightarrow 0
$$

is then an exact sequence of $\widehat{\mathrm{ZH}}$-modules. 
Let $\xi: Y \rightarrow Z_{1}$ be the isomorphism with $\xi\left(x_{i}\right)=z_{i} \forall i$, and let $\nu_{0}: \hat{Y}_{0} \rightarrow \hat{Z}_{2}$ be the $\widehat{\mathbf{Z H}}$-homomorphism given by

$$
\nu_{0}\left(x_{i}\right)=\sum_{j>i}\left(\mathscr{M}_{i j} /\left(t_{i}-1\right)\right) z_{i j}-\sum_{j<i}\left(\mathscr{M}_{i j} /\left(t_{i}-1\right)\right) z_{j i} ;
$$

we denote by $\mathscr{N}$ the matrix of $\nu_{0}$. For $1 \leqslant r \leqslant \mu$ let $\nu_{r}: \hat{Y}_{r} \rightarrow \hat{Z}_{2}$ be the $\widehat{\mathbf{Z H}}$-homomorphism whose matrix is obtained from $\mathscr{N}$ by deleting its $r$ th row; if we regard $\hat{Y}_{r}$ as a submodule of $\hat{Y}_{0}$ in the natural way, then $\nu_{r}$ is simply the restriction of $\nu_{0}$ to $\hat{Y}_{r}$. Also, let $\tau: \hat{Z}_{2} \rightarrow \hat{B}_{L}$ be the $\widehat{\mathbf{Z H}}$-homomorphism with $\tau\left(z_{i j}\right)=h\left(\eta\left(\left[x_{i}, x_{j}\right]\right) G^{\prime \prime}\right)$. For $0 \leqslant r \leqslant \mu$ we now have the following diagram of $\widehat{\mathbf{Z H}}$-modules and $\overline{\mathrm{ZH}}$-homomorphisms:

$\begin{array}{ccccc}\hat{Y}_{r} \oplus \hat{Z}_{3} & & \hat{Y}_{r} & & \hat{Z}_{2} \\ \left\langle v_{r}, \hat{\zeta}_{3}\right\rangle \downarrow & & e_{r} \downarrow & & \downarrow \hat{\zeta}_{2} \\ \hat{Z}_{2} & \stackrel{\hat{\xi}^{-1} \hat{\zeta}_{2}}{\rightarrow} & \hat{Y} & \stackrel{\hat{\xi}}{\rightarrow} & \hat{Z}_{1} \\ \tau \downarrow & & f \downarrow & & \downarrow \hat{\zeta}_{1} \\ \hat{B}_{L} & \stackrel{\hat{\phi}}{\rightarrow} & \hat{A}_{L} & \stackrel{\psi}{\rightarrow} & \widehat{I H} \\ \downarrow & & \downarrow & & \downarrow \\ 0 & & 0 & & 0\end{array}$

THEOREM (3.5). This diagram commutes and has exact columns. Consequently, the matrix

$$
\mathscr{P}=\left(\begin{array}{c}
\mathscr{N} \\
N_{3}(\mu)
\end{array}\right)
$$

is a presentation matrix for the $\widehat{\mathbf{Z H}}$-module $\hat{B}_{L}$, as is the submatrix of $\mathscr{P}$ obtained by deleting any one of its first $\mu$ rows.

Proof. If $1 \leqslant i<j \leqslant \mu$ then

$$
\begin{aligned}
f \hat{\xi}^{-1} \hat{\zeta}_{2}\left(z_{i j}\right) & =f\left(\left(t_{i}-1\right) x_{j}-\left(t_{j}-1\right) x_{i}\right) \\
& =h\left(1 \otimes \eta\left(\left(x_{i}-1\right)\left(x_{j}-1\right)-\left(x_{j}-1\right)\left(x_{i}-1\right)\right)\right) \\
& =h\left(1 \otimes \eta\left(x_{i} x_{j}\right)\left(1-\eta\left(x_{j}^{-1} x_{i}^{-1} x_{j} x_{i}\right)\right)\right) \\
& =-t_{i} t_{j} \cdot h \phi\left(\eta\left(x_{j}^{-1} x_{i}^{-1} x_{j} x_{i}\right) G^{\prime \prime}\right) \\
& =-h \phi\left(\eta\left(x_{i} x_{j} \cdot x_{j}^{-1} x_{i}^{-1} x_{j} x_{i} \cdot x_{j}^{-1} x_{i}^{-1}\right) G^{\prime \prime}\right) \\
& =-\hat{\phi} h\left(\eta\left(x_{j} x_{i} x_{j}^{-1} x_{i}^{-1}\right) G^{\prime \prime}\right)=\hat{\phi} \tau\left(z_{i j}\right),
\end{aligned}
$$

so the diagram does indeed commute. 
In addition, note that for $1 \leqslant i \leqslant \mu, \sum_{j=1}^{\mu} \mathscr{M}_{i j} \cdot\left(t_{j}-1\right)=0$, and hence

$$
\begin{aligned}
\hat{\zeta}_{2} \nu_{0}\left(x_{i}\right) & =\sum_{j \neq i} \mathscr{M}_{i j} z_{j}-\sum_{j \neq i}\left(\mathscr{M}_{i j} /\left(t_{i}-1\right)\right)\left(t_{j}-1\right) z_{i} \\
& =\sum_{j=1}^{\mu} \mathscr{M}_{i j} z_{j}=\hat{\xi} e_{0}\left(x_{i}\right),
\end{aligned}
$$

so that $\hat{\zeta}_{2} \nu_{0}=\hat{\xi} e_{0}$; it follows also that $\hat{\zeta}_{2} \nu_{r}=\hat{\xi} e_{r}$ for $1 \leqslant r \leqslant \mu$.

A straightforward diagram-chase completes the proof. Q.E.D.

4. Elementary ideals. Recall that if $m, n \geqslant 1$ and $M$ is an $m \times n$ matrix with entries in a commutative ring $R$ with unity, then its elementary (or determinantal) ideals, denoted $E_{k}(M)$ and indexed by $k \in \mathbf{Z}$, are ideals of $R$ defined by: if $k<n-m$ or $k<0$ then $E_{k}(M)=0$; if $k \geqslant n$ then $E_{k}(M)=R$; and if $0, n-m \leqslant$ $k<n$ then $E_{k}(M)$ is the ideal of $R$ generated by the determinants of the $(n-k) \times$ $(n-k)$ submatrices of $M$. If $M$ is a presentation matrix for an $R$-module $A$, then the elementary ideals (or Fitting invariants) of $A$ are defined by $E_{k}(A)=E_{k}(M)$; these ideals depend only on the isomorphism-type of $A$, and are independent of the choice of a particular presentation matrix $M[17$, p. 58]. If $f: R \rightarrow S$ is a homomorphism of commutative rings with unity, and we consider $S$ as an $R$-module via $f$, then it follows from the right exactness of tensor products that $f(M)$ is a presentation matrix for the tensor product $S \otimes_{R} A$, considered as an $S$-module with the scalar multiplication performed in the first factor. Consequently, for $k \in \mathbf{Z}$, $E_{k}\left(S \otimes_{R} A\right)$ is the ideal of $S$ generated by $f\left(E_{k}(A)\right)$.

Considering the inclusion $\mathbf{Z} H \subseteq \widehat{\mathbf{Z H}}$, we conclude that if $A$ is a finitely presented $\mathbf{Z} H$-module then for $k \in \mathbf{Z}$ the ideal of $\widehat{\mathbf{Z H}}$ generated by $E_{k}(A)$ is $E_{k}\left(\widehat{\mathbf{Z H}} \otimes_{\mathbf{Z} H} A\right)$; since $\widehat{\mathbf{Z H}} \otimes_{\mathrm{Z} H} A$ is isomorphic to $\hat{A}\left[1\right.$, III, §3.4], this ideal is $E_{k}(\hat{A})$. In view of Theorems (3.4) and (3.5), we conclude

THEOREM( 4.1.). For any $k \in \mathbf{Z}$, the ideal of $\widehat{\mathbf{Z H}}$ generated by $E_{k}\left(A_{L}\right)$ is $E_{k}(\mathscr{M})$, and this remains true if any row of $\mathscr{M}$ is deleted. Also, the ideal of $\widehat{\mathbf{Z H}}$ generated by $E_{k}\left(B_{L}\right)$ is $E_{k}(\mathscr{P})$, and this remains true if any one of the first $\mu$ rows of $\mathscr{P}$ is deleted.

In practice, the matrices $\mathscr{M}$ and $\mathscr{P}$ may be rather inconvenient, since determining their entries requires the determination of all the (infinitely many) integers $\mu\left(i_{1}, \ldots, i_{q}\right)$; for this reason we state several results which are weaker than Theorem (4.1) but involve only the simpler matrices $\mathscr{M}_{q}$ and $\mathscr{P}_{q}$.

THEOREM (4.2). If $0<k<\mu$ and $q \geqslant 1$, then

$$
\sum_{i=0}^{k-1} E_{\mu-k+i}\left(A_{L}\right) \cdot(I H)^{q i}+(I H)^{q k}=\sum_{i=0}^{k-1} E_{\mu-k+i}\left(\mathscr{M}_{q}\right) \cdot(I H)^{q i}+(I H)^{q k},
$$

and this remains true if any row of $\mathscr{M}_{q}$ is deleted.

Proof. Clearly any presentation matrix of $A_{L}$ may be transformed into a presentation matrix of $A_{L} /(I H)^{q} \cdot A_{L}$ by the adjunction of a number of rows whose 
entries all lie in $(I H)^{q}$. By [22, Lemma (3.1)], then,

$$
\sum_{i \geqslant 0} E_{\mu-k+i}\left(A_{L}\right) \cdot(I H)^{q i}=\sum_{i \geqslant 0} E_{\mu-k+i}\left(A_{L} /(I H)^{q} \cdot A_{L}\right) \cdot(I H)^{q i}
$$

for any $k \in \mathbf{Z}$. Combining Propositions (3.2) and (3.3), we note that $\mathscr{M}_{q}$ can be transformed into a presentation matrix of $A_{L} /(I H)^{q} \cdot A_{L}$ by the adjunction of a number of rows all of whose entries lie in $(I H)^{q}$, so that for $k \in \mathbf{Z}$

$$
\sum_{i \geqslant 0} E_{\mu-k+i}\left(A_{L}\right) \cdot(I H)^{q i}=\sum_{i \geqslant 0} E_{\mu-k+i}\left(\mathscr{M}_{q}\right) \cdot(I H)^{q i} .
$$

In particular,

$$
\sum_{i \geqslant 0} E_{\mu+i}\left(A_{L}\right) \cdot(I H)^{q i}=\sum_{i \geqslant 0} E_{\mu+i}\left(\mathscr{M}_{q}\right) \cdot(I H)^{q i}=\mathbf{Z} H
$$

so for $0<k<\mu$

$$
\begin{aligned}
\sum_{i=0}^{k-1} E_{\mu-k+i}\left(A_{L}\right) \cdot(I H)^{q i}+(I H)^{q k} & =\sum_{i \geqslant 0} E_{\mu-k+i}\left(A_{L}\right) \cdot(I H)^{q i} \\
& =\sum_{i \geqslant 0} E_{\mu-k+i}\left(\mathscr{M}_{q}\right) \cdot(I H)^{q i} \\
& =\sum_{i=0}^{k-1} E_{\mu-k+i}\left(\mathscr{M}_{q}\right) \cdot(I H)^{q i}+(I H)^{q k} .
\end{aligned}
$$

By Proposition (3.3), this remains true if any row of $\mathscr{M}_{q}$ is deleted. Q.E.D.

The case $q=1$ of this result is essentially the assertion that $E_{\mu-j}\left(A_{L}\right) \subseteq(I H)^{j}$ for $0<j<\mu$, which was observed by R. H. Fox [8, p. 209]. The case $q=2$ is also already known [22, Theorem 1]. Since $E_{\mu-j}\left(\mathscr{M}_{q}\right)$ and $E_{\mu-j}\left(A_{L}\right)$ are both contained in $(I H)^{j}$ for $0<j<\mu$, it is a simple matter to derive

Corollary (4.3). For $0<k<\mu$ and $q \geqslant 1$,

$$
E_{\mu-k}\left(A_{L}\right)+(I H)^{k+q-1}=E_{\mu-k}\left(\mathscr{M}_{q}\right)+(I H)^{k+q-1},
$$

and this remains true if any row of $\mathscr{M}_{q}$ is deleted.

For $q \geqslant 1$ let $\mathscr{P}_{q}$ be the matrix obtained from $\mathscr{P}$ by ignoring those summands in the definition of the entries of $\mathscr{P}$ which are of degree $\geqslant q$ as monomials in $t_{1}-1, \ldots, t_{\mu}-1$. That is, $\mathscr{P}_{q}$ is related to $\mathscr{P}$ just as $\mathscr{M}_{q}$ is related to $\mathscr{M}$. In analogy with Theorem (4.2), we have

THEOREM (4.4). If $0<k \leqslant\left(\begin{array}{c}\mu \\ 2\end{array}\right)$,

$$
\sum_{i=0}^{k-1} E_{\left(\frac{\mu}{2}\right)-k+i}\left(B_{L}\right) \cdot(I H)^{q i}+(I H)^{q k}=\sum_{i=0}^{k-1} E_{\left(\frac{\mu}{2}\right)-k+i}\left(\mathscr{P}_{q}\right) \cdot(I H)^{q i}+(I H)^{q k},
$$

and this remains true if any of the first $\mu$ rows of $\mathscr{P}_{q}$ is deleted.

Proof. Any presentation matrix of $B_{L}$ can be transformed into a presentation matrix of $B_{L} /(I H)^{q} \cdot B_{L}$ by adjoining a number of rows whose entries all lie in $(I H)^{q}$. By $[22$, Lemma (3.1)],

$$
\sum_{i \geqslant 0} E_{\left(\frac{\mu}{2}\right)-k+i}\left(B_{L}\right) \cdot(I H)^{q i}=\sum_{i \geqslant 0} E_{\left(\frac{\mu}{2}\right)-k+i}\left(B_{L} /(I H)^{q} \cdot B_{L}\right) \cdot(I H)^{q i}
$$


for all $k \in \mathbf{Z}$. Since $h: B_{L} \rightarrow \hat{B}_{L}$ induces an isomorphism $B_{L} /(I H)^{q} \cdot B_{L} \rightarrow$ $\hat{B}_{L} /(\widehat{I H})^{q} \cdot \hat{B}_{L}\left[1\right.$, III, §2.12] and $\mathscr{P}$ is a presentation matrix for $\hat{B}_{L}$, a presentation matrix of $B_{L} /(I H)^{q} \cdot B_{L}$ can be obtained from $\mathscr{P}_{q}$ by adjoining some rows whose entries all lie in $(I H)^{q}$, so by another application of [22, Lemma (3.1)]

$$
\sum_{i \geqslant 0} E_{\left(\frac{\mu}{2}\right)-k+i}\left(B_{L}\right) \cdot(I H)^{q i}=\sum_{i \geqslant 0} E_{\left(\frac{\mu}{2}\right)-k+i}\left(\mathscr{P}_{q}\right) \cdot(I H)^{q i}
$$

for every $k \in \mathbf{Z}$.

The proof proceeds in the same manner as that of Theorem (4.2). Q.E.D.

The matrix $\mathscr{P}_{1}$ is simply $\varepsilon(\mathscr{N})$ with $\left(\begin{array}{l}\mu \\ 3\end{array}\right)$ rows of zeros adjoined, so when $q=1$ Theorem (4.4) remains true if $\mathscr{P}_{1}$ is replaced by $\varepsilon(\mathscr{N})$. In this form, the $q=1$ case of Theorem (4.4) is already known [23, Theorem 2].

In analogy with Corollary (4.3), we have

COROLlaRY (4.5). If $0<k<\mu$ and $q \geqslant 1$, then

$$
E_{\left(\frac{\mu}{2}\right)-k}\left(B_{L}\right)+(I H)^{q}=E_{\left(\frac{\mu}{2}\right)-k}\left(\mathscr{P}_{q}\right)+(I H)^{q} .
$$

Also, if $\mu \leqslant k \leqslant\left(\begin{array}{c}\mu \\ 2\end{array}\right)$ and $q \geqslant 1$, then

$$
E_{\left(\frac{\mu}{2}\right)-k}\left(B_{L}\right)+(I H)^{q+k-\mu}=E_{\left(\frac{\mu}{2}\right)-k}\left(\mathscr{P}_{q}\right)+(I H)^{q+k-\mu} .
$$

Both statements remain true if any one of the first $\mu$ rows of $\mathscr{P}_{q}$ is deleted.

Proof. The first equality of the statement follows immediately from Theorem (4.4).

The rows of the $\mu \times\left(\frac{\mu}{2}\right)$ matrix $\varepsilon(\mathscr{N})$ are linearly dependent (in fact, their sum is zero), so $E_{\left(\frac{\mu}{2}\right)-\mu}(\varepsilon(\mathscr{N}))=0$. From this and the modified form of Theorem (4.4) just mentioned, it follows that $E_{\left(\mu_{2}^{-1}\right)-j}\left(B_{L}\right) \subseteq(I H)^{j}$ for $0 \leqslant j \leqslant\left(\begin{array}{c}\mu_{2}-1 \\ 2\end{array}\right)$. This and Theorem (4.4) imply that also $E_{\left(\mu_{2}-1\right)-j}\left(\mathscr{P}_{q}\right) \subseteq(I H)^{j}$ for $0 \leqslant j \leqslant\left(\begin{array}{c}\mu-1 \\ 2\end{array}\right)$. Combining these inclusions with Theorem (4.4) yields the second equality of the statement. Q.E.D.

5. The Alexander polynomials of links with all linking numbers zero. A couple of ring-theoretic lemmas will be useful.

LEMMA (5.1). Let $m \geqslant 1$, and let $\mathbf{Z}\left[u_{1}, \ldots, u_{m}, u_{1}^{-1}, \ldots, u_{m}^{-1}\right]$ be the ring of Laurent polynomials (with integer coefficients) in the $m$ commuting indeterminates $u_{1}, \ldots, u_{m}$. If $I$ is the ideal of $\mathbf{Z}\left[u_{1}, \ldots, u_{m}, u_{1}^{-1}, \ldots, u_{m}^{-1}\right]$ generated by $\left\{u_{1}-1, \ldots, u_{m}-1\right\}$, then for $a, b \in \mathbf{Z}\left[u_{1}, \ldots, u_{m}, u_{1}^{-1}, \ldots, u_{m}^{-1}\right]$ and $k \geqslant 0, a b \in I^{k}$ iff there is $a j \in\{0, \ldots, k\}$ with $a \in I^{j}$ and $b \in I^{k-j}$.

Proof. This is easily verified using the fact that, for $j \geqslant 2, I^{j}$ consists of those elements of $I$ whose partial derivatives (in the usual sense of multivariate calculus) of orders $<j$ all vanish at the point $u_{1}=1, \ldots, u_{m}=1$. Q.E.D.

Note that if $m=\mu$, there is an isomorphism between $\mathbf{Z}\left[u_{1}, \ldots, u_{m}, u_{1}^{-1}, \ldots, u_{m}^{-1}\right]$ and $\mathbf{Z} H$ under which each $u_{i}$ corresponds to $t_{i}$, and (hence) $I$ corresponds to $I H$.

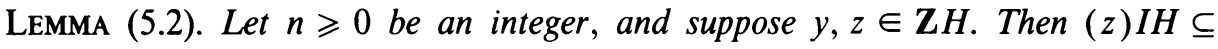
(y) $I H+(I H)^{n+1}$ iff $z \in(y)+(I H)^{n}$.

Proof. Certainly if $z \in(y)+(I H)^{n}$ then $(z) I H \subseteq(y) I H+(I H)^{n+1}$. 
Conversely, suppose $(z) I H \subseteq(y) I H+(I H)^{n+1}$. If $y \in(I H)^{n}$, then $z \in(I H)^{n}$.

On the other hand, suppose $y \notin(I H)^{n}$; then there is a unique $r \in\{0, \ldots, n-1\}$ such that $y \in(I H)^{r}-(I H)^{r+1}$. Since $(z) I H \subseteq(y) I H+(I H)^{n+1}$, whenever $1 \leqslant i$ $\leqslant \mu$ there are $a_{i 1}, \ldots, a_{i \mu} \in \mathbf{Z} H$ and $w_{i} \in(I H)^{n+1}$ with

$$
z \cdot\left(t_{i}-1\right)=w_{i}+\sum_{j=1}^{\mu} a_{i j} y\left(t_{j}-1\right) .
$$

We claim that whenever $1 \leqslant i, k \leqslant \mu$ there are $a_{i 1 k}, \ldots, a_{i \mu k} \in \mathbf{Z} H$ and $w_{i k} \in$ $(I H)^{n+1}$ such that

$$
z \cdot\left(t_{i}-1\right)=w_{i k}+a_{i i k} y\left(t_{i}-1\right)+\sum_{i \neq j \geqslant k} a_{i j k} y\left(t_{j}-1\right) .
$$

This claim is verified by induction on $k$; if $k=1$, it is clearly true.

Suppose, now, that $k \geqslant 2$ and the claim is satisfied by $k-1$ and every $i \in$ $\{1, \ldots, \mu\}$. If $i=k-1$, the claim is immediately satisfied by $k$ and $i$.

If $i \neq k-1$, then

$$
\begin{aligned}
v= & w_{k-1 k-1}\left(t_{i}-1\right)-w_{i k-1}\left(t_{k-1}-1\right) \\
= & a_{i i k-1} y\left(t_{i}-1\right)\left(t_{k-1}-1\right)+\sum_{i \neq j \geqslant k-1} a_{i j k-1} y\left(t_{j}-1\right)\left(t_{k-1}-1\right) \\
& -a_{k-1 k-1 k-1} y\left(t_{k-1}-1\right)\left(t_{i}-1\right)-\sum_{j \geqslant k} a_{k-1 j k-1} y\left(t_{j}-1\right)\left(t_{i}-1\right)
\end{aligned}
$$

is an element of $(I H)^{n+2}$. Since $y \in(I H)^{r}-(I H)^{r+1}$, then, $v / y \in(I H)^{n+2-r}$.

Let $m=k-2$ or $k-1$ according to whether $i<k-1$ or $i>k-1$. For $1 \leqslant j \leqslant m$ let $i_{j}=j$ if $j<i$, and $i_{j}=j+1$ if $j \geqslant i$, so that $i_{1}, \ldots, i_{m}$ are the integers strictly less than $k$ and not equal to $i$, in ascending order. Let $\phi: \mathbf{Z} H \rightarrow$ $\mathbf{Z}\left[u_{1}, \ldots, u_{m}, u_{1}^{-1}, \ldots, u_{m}^{-1}\right]$ be the homomorphism of rings with unity given by $\phi\left(t_{i_{j}}\right)=u_{j}$ for $1 \leqslant j \leqslant m$, and $\phi\left(t_{s}\right)=1$ for $s \notin\left\{i_{1}, \ldots, i_{m}\right\}$. Then $\phi(v / y)=$ $\phi\left(a_{i k-1 k-1}\right)\left(u_{m}-1\right)^{2} \in \phi(I H)^{n+2-r}$, so $\phi\left(a_{i k-1 k-1}\right) \in \phi(I H)^{n-r}$. Then $a_{i k-1 k-1}$ $\in(I H)^{n-r}+\operatorname{ker} \phi$, so

$$
a_{i k-1 k-1}=w^{\prime}+a_{i}\left(t_{i}-1\right)+\sum_{i \neq j \geqslant k} a_{j}\left(t_{j}-1\right)
$$

for some $a_{i}, a_{k}, \ldots, a_{\mu} \in \mathbf{Z} H$ and $w^{\prime} \in(I H)^{n-r}$. Then

$$
z \cdot\left(t_{i}-1\right)=w_{i k}+a_{i i k} y\left(t_{i}-1\right)+\sum_{i \neq j \geqslant k} a_{i j k} y\left(t_{j}-1\right),
$$

where $w_{i k}=w_{i k-1}+w^{\prime} y\left(t_{k-1}-1\right) \in(I H)^{n+1}$, and for $j=i$ or $j \geqslant k, a_{i j k}=a_{i j k-1}$ $+a_{j}\left(t_{k-1}-1\right)$. This completes the proof of the claim.

By the claim, $z\left(t_{\mu}-1\right)=w_{\mu \mu}+a y\left(t_{\mu}-1\right)$ for some $a=a_{\mu \mu \mu} \in \mathbf{Z} H$ and $w_{\mu \mu} \in$ $(I H)^{n+1}$. If $w=z-a y$, then $w\left(t_{\mu}-1\right)=w_{\mu \mu} \in(I H)^{n+1}$, so $w \in(I H)^{n}$. Then $z=w+a y \in(I H)^{n}+(y)$. Q.E.D.

TheOREM (5.3). Let $L=K_{1} \cup \cdots \cup K_{\mu} \subseteq S^{3}$ be a tame link with $\mu(i, j)=0$ $\forall i \neq j \in\{1, \ldots, \mu\}$. Then $\Delta_{1}\left(A_{L}\right) \in(I H)^{2 \mu-2}$ if $\mu$ is even, while if $\mu$ is odd there is an $x \in(I H)^{(\mu-3) / 2}$ with

$$
\left(\Delta_{1}\left(A_{L}\right)\right)+(I H)^{2 \mu-2}=\left(x^{2} \cdot \prod_{i=1}^{\mu}\left(t_{i}-1\right)\right)+(I H)^{2 \mu-2} .
$$


Proof. Let $\mathscr{M}_{3}^{\prime}$ be the matrix obtained from $\mathscr{M}_{3}$ by deleting its last row. By Theorem (4.2),

$$
\sum_{i=0}^{\mu-2} E_{1+i}\left(A_{L}\right) \cdot(I H)^{3 i}+(I H)^{3(\mu-1)}=\sum_{i=0}^{\mu-2} E_{1+i}\left(\mathscr{M}_{3}^{\prime}\right) \cdot(I H)^{3 i}+(I H)^{3(\mu-1)} .
$$

Since $\mu(i, j)=0 \forall i \neq j \in\{1, \ldots, \mu\}$, every entry of $\mathscr{M}_{3}^{\prime}$ is in $(I H)^{2}$, so $E_{1+i}\left(\mathscr{M}_{3}^{\prime}\right)$ $\subseteq(I H)^{2(\mu-1-i)}$ for $0 \leqslant i \leqslant \mu-2$; it follows from Theorem (4.2) that also $E_{1+i}\left(A_{L}\right) \subseteq(I H)^{2(\mu-1-i)}$ for $0 \leqslant i \leqslant \mu-2$. Hence adding $(I H)^{2 \mu-1}$ to each side of the equality above yields

$$
E_{1}\left(A_{L}\right)+(I H)^{2 \mu-1}=E_{1}\left(\mathscr{M}_{3}^{\prime}\right)+(I H)^{2 \mu-1} .
$$

Consider the ideal $E_{1}\left(\mathscr{M}_{3}^{\prime}\right) \subseteq \mathbf{Z} H$. It is generated by the determinants of the $\mu$ square submatrices of $\mathscr{M}_{3}^{\prime}$ of order $\mu-1$. Since $\sum_{j}\left(\mathscr{M}_{3}^{\prime}\right)_{i j}\left(t_{j}-1\right)=0$ whenever $1 \leqslant i \leqslant \mu-1$, a simple argument shows that there is a single element $\Delta \in \mathbf{Z} H$ such that these $\mu$ determinants are $\Delta \cdot\left(t_{1}-1\right), \ldots, \Delta \cdot\left(t_{\mu}-1\right)$, up to sign. Then $E_{1}\left(\mathscr{M}_{3}^{\prime}\right)$ $=(\Delta) \cdot I H$, so since $E_{1}\left(A_{L}\right)=\left(\Delta_{1}\left(A_{L}\right)\right) \cdot I H$ we may deduce from Lemma $(5.2)$ that

$$
\left(\Delta_{1}\left(A_{L}\right)\right)+(I H)^{2 \mu-2}=(\Delta)+(I H)^{2 \mu-2} .
$$

Let $\mathscr{M}_{3}^{\prime \prime}$ be the submatrix of $\mathscr{M}_{3}^{\prime}$ obtained by deleting its last column; then $\Delta \cdot\left(t_{\mu}-1\right)= \pm \operatorname{det} \mathscr{M}_{3}^{\prime \prime}$, and we may as well assume that $\Delta \cdot\left(t_{\mu}-1\right)=\operatorname{det} \mathscr{M}_{3}^{\prime \prime}$.

Since $\mu(i, j)=0 \forall i, j \in\{1, \ldots, \mu\}$,

$$
\Delta(i, j, k)=0 \quad \forall i, j, k \in\{1, \ldots, \mu\},
$$

and hence by cyclic symmetry [14, Theorem 6] $\mu(i, j, k)=\mu(k, i, j) \forall i, j, k \in$ $\{1, \ldots, \mu\}$. Also, $\mu(i, j, k)=-\mu(j, i, k) \forall i, j, k \in\{1, \ldots, \mu\}$ [14, Theorem 6], and consequently $\mu(i, j, k)=0$ whenever any two of $i, j, k$ coincide.

It follows that if $i \neq j \in\{1, \ldots, \mu-1\}$ then the $i j$ entry of $\mathscr{M}_{3}^{\prime \prime}$ is

$$
\left(\mathscr{M}_{3}\right)_{i j}=\sum_{i \neq k \neq j} \mu(k, j, i)\left(t_{i}-1\right)\left(t_{k}-1\right) .
$$

Also, if $i \in\{1, \ldots, \mu-1\}$ the $i i$ entry of $\mathscr{M}_{3}^{\prime \prime}$ is

$$
\left(\mathscr{M}_{3}\right)_{i i}=-\sum_{i \neq j \neq k \neq i} \mu(k, j, i)\left(t_{k}-1\right)\left(t_{j}-1\right),
$$

and since $\mu(k, j, i)=-\mu(j, k, i) \forall i, j, k \in\{1, \ldots, \mu\}$ this sum is 0 . Note that in general every entry of the $i$ th row of $\mathscr{M}_{3}^{\prime \prime}$ is divisible by $t_{i}-1$.

If $Y$ is the matrix obtained from $\mathscr{M}_{3}^{\prime \prime}$ by dividing its $i$ th row by $t_{i}-1$ for each $i$, then

$$
\operatorname{det} Y \cdot \prod_{i=1}^{\mu-1}\left(t_{i}-1\right)=\operatorname{det} \mathscr{M}_{3}^{\prime \prime}
$$


The diagonal entries of $Y$ are all 0 , while if $i \neq j \in\{1, \ldots, \mu-1\}$ the $i j$ entry of $Y$ is

$$
\sum_{i \neq k \neq j} \mu(k, j, i)\left(t_{k}-1\right)
$$

since $\mu(k, j, i)=-\mu(k, i, j) \forall i, j, k \in\{1, \ldots, \mu\}$, this is the negative of the $j i$ entry of $Y$. Thus $Y$ is a skew-symmetric $(\mu-1) \times(\mu-1)$ matrix, so either det $Y=0$ (if $\mu-1$ is odd) or there is a $y \in \mathbf{Z} H$ with det $Y=y^{2}$ (if $\mu-1$ is even) (see, for instance, [10, p. 334]).

If $\mu$ is even, then, $\operatorname{det} Y=0$, so $\Delta=0$.

On the other hand, suppose $\mu$ is odd. Then $y^{2} \cdot \prod_{i<\mu}\left(t_{i}-1\right)=\operatorname{det} \mathscr{M}_{3}^{\prime \prime}=\Delta \cdot\left(t_{\mu}\right.$ - 1), so since $t_{\mu}-1 \in \mathbf{Z} H$ is a prime, $y=x\left(t_{\mu}-1\right)$ for some $x \in \mathbf{Z} H$; by cancellation, then,

$$
\Delta=x^{2} \cdot \prod_{i=1}^{\mu}\left(t_{i}-1\right) .
$$

Note that since every entry of $Y$ lies in $I H$, necessarily $x^{2}\left(t_{\mu}-1\right)^{2}=\operatorname{det} Y \in$ $(I H)^{\mu-1}$, so $x$ must be an element of $(I H)^{(\mu-3) / 2}$. Q.E.D.

Incidentally, for even $\mu$ the proof can be considerably shortened by using Proposition (6.1). Also, we should remark that Theorem (5.3) is best possible, in the sense that it is possible to have $\Delta_{1}\left(A_{L}\right) \notin(I H)^{2 \mu-1}$ if $\mu$ is even, and $\Delta_{1}\left(A_{L}\right) \notin$ $(I H)^{2 \mu-2}$ if $\mu$ is odd. If $\mu=2 \nu$, such an example is the connected sum of $\nu-1$ copies of the Borromean rings $\left(6_{2}^{3}\right.$ in [18, Appendix C]) and one copy of Whitehead's link $\left(5_{1}^{2}\right.$ in [18, Appendix C]); if $\mu=2 v+1$, such an example is the connected sum of $\nu$ copies of the Borromean rings.

6. Links of two components. Before restricting our attention to the special case $\mu=2$, we state an interesting consequence of the symmetry of the Alexander polynomials of links, first noted (for $\mu=2$ ) by W. S. Massey [12, 2 , condition (a)].

Proposition (6.1). Let $L \subseteq S^{3}$ be a tame link of $\mu \geqslant 2$ components. If $\Delta_{1}\left(A_{L}\right) \in$ $(I H)^{\mu+2 n-1}$, then $\Delta_{1}\left(A_{L}\right) \in(I H)^{\mu+2 n}$.

Proof. The ring $\mathbf{Z} H$ possesses an involution, denoted by an overbar, defined by $\bar{t}_{i}=t_{i}^{-1}$. If $\Delta_{1}\left(A_{L}\right) \in(I H)^{\mu+2 n-1}$ then, by Torres' first relation [20], $\Delta_{1}\left(A_{L}\right)-$ $(-1)^{\mu} \overline{\Delta_{1}\left(A_{L}\right)} \in(I H)^{\mu+2 n}$.

If $r_{1}, \ldots, r_{\mu} \geqslant 0$ have $\sum r_{i}=\mu+2 n-1$, then clearly for any $m \in \mathbf{Z}$ the monomial $y=m \cdot \Pi\left(t_{i}-1\right)^{r_{i}}$ has the property that $y-(-1)^{\mu+2 n-1} \cdot \bar{y} \in(I H)^{\mu+2 n}$. Since $\Delta_{1}\left(A_{L}\right) \in(I H)^{\mu+2 n-1}, \Delta_{1}\left(A_{L}\right)$ is congruent (modulo $\left.(I H)^{\mu+2 n}\right)$ to a sum of such monomials, and hence $\Delta_{1}\left(A_{L}\right)-(-1)^{\mu+2 n-1} \cdot \overline{\Delta_{1}\left(A_{L}\right)} \in(I H)^{\mu+2 n}$.

Thus $\Delta_{1}\left(A_{L}\right)+\Delta_{1}\left(A_{L}\right) \in(I H)^{\mu+2 n}$, so $\Delta_{1}\left(A_{L}\right) \in(I H)^{\mu+2 n}$. Q.E.D.

For the remainder of this section we will assume, unless stated otherwise, that $L \subseteq S^{3}$ is a tame link of $\mu=2$ components.

For integers $v \geqslant 1$ and $w \geqslant 0$ let $C(v, w)$ be the set of all $(v+w)$-tuples $\left(c_{1}, \ldots, c_{v^{+}+w}\right)$ in which 1 appears $v$ times and 2 appears $w$ times. 
LEMMA (6.2). For any integer $u \geqslant 1$,

$$
\begin{aligned}
& \sum_{C(v, w)} \mu\left(c_{1}, \ldots, c_{v+w}, 2_{1}, \ldots, 2_{u}, 1\right) \\
& \quad=\sum_{i=0}^{w}(-1)^{i}\left(\begin{array}{c}
u-1+i \\
i
\end{array}\right)\left(\begin{array}{c}
\mu(2,1)-i-u \\
w-i
\end{array}\right) \mu\left(1_{1}, \ldots, 1_{v}, 2_{1}, \ldots, 2_{u+i}, 1\right),
\end{aligned}
$$

where $1_{j}=1$ and $2_{j}=2 \forall j$.

Proof. Suppose, first, that $w=0$. Then $C(v, w)$ has only one element, $\left(1_{1}, \ldots, 1_{v}\right)$, so the first of the two sums is $\mu\left(1_{1}, \ldots, 1_{v}, 2_{1}, \ldots, 2_{u}, 1\right)$. The second sum is the same.

Proceeding inductively, suppose $w \geqslant 1$. Define functions $f_{j}: C(v, w-1) \rightarrow$ $C(v, w), \quad 1 \leqslant j \leqslant v+w$, by $f_{j}\left(c_{1}, \ldots, c_{v+w-1}\right)=\left(c_{1}, \ldots, c_{j-1}, 2, c_{j}, \ldots, c_{v+w-1}\right)$. Clearly then each $f_{j}$ is injective, and each element of $C(v, w)$ is in the image of precisely $w$ of the $f_{j}$. Hence

$$
\begin{aligned}
w \cdot & \sum_{C(v, w)} \mu\left(c_{1}, \ldots, c_{v+w}, 2_{1}, \ldots, 2_{u}, 1\right) \\
\quad= & \sum_{C(v, w-1)} \sum_{j=1}^{v+w} \mu\left(f_{j}\left(c_{1}, \ldots, c_{v+w-1}\right), 2_{1}, \ldots, 2_{u}, 1\right) .
\end{aligned}
$$

If $\left(c_{1}, \ldots, c_{v+w-1}\right) \in C(v, w-1)$, then (in the terminology of [2]) the sequences $\left(c_{1}, \ldots, c_{v+w-1}, 2_{1}, \ldots, 2_{u}\right)$ and (2) have $2 u+v+2 w-1$ infiltrations: $u+w-1$ with result $\left(c_{1}, \ldots, c_{v+w-1}, 2_{1}, \ldots, 2_{u}\right), u$ with result $\left(c_{1}, \ldots, c_{v+w-1}, 2_{1}, \ldots, 2_{u+1}\right)$, and one with result $\left(f_{j}\left(c_{1}, \ldots, c_{v+w-1}\right), 2_{1}, \ldots, 2_{u}\right)$ for each $j \in\{1, \ldots, v+w\}$. By [2, Lemma (3.3)], then,

$$
\begin{aligned}
w \cdot & \sum_{C(v, w)} \mu\left(c_{1}, \ldots, c_{v+w}, 2_{1}, \ldots, 2_{u}, 1\right) \\
= & \sum_{C(v, w-1)}(\mu(2,1)-u-w+1) \mu\left(c_{1}, \ldots, c_{v+w-1}, 2_{1}, \ldots, 2_{u}, 1\right) \\
& \quad-\sum_{C(v, w-1)} u \cdot \mu\left(c_{1}, \ldots, c_{v+w-1}, 2_{1}, \ldots, 2_{u+1}, 1\right) .
\end{aligned}
$$

Applying the inductive hypothesis, this is equal to

$$
\begin{aligned}
\sum_{i=0}^{w-1}( & -1)^{i}\left(\begin{array}{c}
u-1+i \\
i
\end{array}\right)(\mu(2,1)-u-w+1) \\
& \times\left(\begin{array}{c}
\mu(2,1)-i-u \\
w-i-1
\end{array}\right) \mu\left(1_{1}, \ldots, 1_{v}, 2_{1}, \ldots, 2_{u+i}, 1\right) \\
& +\sum_{i=1}^{w}(-1)^{i} u\left(\begin{array}{c}
u+i-1 \\
i-1
\end{array}\right)\left(\begin{array}{c}
\mu(2,1)-i-u \\
w-i
\end{array}\right) \mu\left(1_{1}, \ldots, 1_{v}, 2_{1}, \ldots, 2_{u+i}, 1\right) \\
& =w \cdot \sum_{i=0}^{w}(-1)^{i}\left(\begin{array}{c}
u-1+i \\
i
\end{array}\right)\left(\begin{array}{c}
\mu(2,1)-i-u \\
w-i
\end{array}\right) \mu\left(1_{1}, \ldots, 1_{v}, 2_{1}, \ldots, 2_{u+i}, 1\right) .
\end{aligned}
$$

(Repeated use has been made of the identity $a\left(\begin{array}{c}a+b \\ b\end{array}\right)=(b+1)\left(\begin{array}{c}a+b \\ b+1\end{array}\right)$.) Q.E.D. 
Taking $u=1$, we immediately conclude

COROLlaRY (6.3). For any integers $v \geqslant 1$ and $w \geqslant 0$,

$$
\sum_{C\left(v, w^{\prime}\right)} \mu\left(c_{1}, \ldots, c_{v+w}, 2,1\right) \equiv(-1)^{w} \mu\left(1_{1}, \ldots, 1_{v}, 2_{1}, \ldots, 2_{w+1}, 1\right)
$$

( modulo $\Delta\left(1_{1}, \ldots, 1_{v}, 2_{1}, \ldots, 2_{w+1}, 1\right)$ ).

Recall that for $r, s \geqslant 0$ we use $[r+1, s+1]$ to denote the $(r+s+2)$-tuple $\left(1_{1}, \ldots, 1_{r+1}, 2_{1}, \ldots, 2_{s+1}\right)$. Using this notation, we have the following result of $\mathrm{N}$. Smythe [19].

THEOREM (6.4). After multiplying the Alexander polynomial $\Delta_{1}\left(A_{L}\right)$ by -1 if necessary, it will be true that

$$
\frac{1}{r ! s !} \varepsilon\left(\frac{d^{r+s}}{d t_{1}^{r} d t_{2}^{s}}\left(\Delta_{1}\left(A_{L}\right)\right)\right) \equiv(-1)^{s} \mu([r+1, s+1])
$$

(modulo $\Delta([r+1, s+1]))$ for all $r, s \geqslant 0$.

Proof. After deleting the second row of the matrix $\mathscr{P}$, what remains is a $1 \times 1$ matrix whose sole entry is $\mathscr{M}_{12} /\left(t_{1}-1\right)$. By Theorem (4.1), this element of $\widehat{\mathbf{Z H}}$ generates the same principal ideal as $\Delta_{1}\left(A_{L}\right)=\Delta_{0}\left(B_{L}\right)$, since $\Delta_{0}\left(B_{L}\right)$ generates the ideal $E_{0}\left(B_{L}\right)$ of $\mathbf{Z} H$. Thus there is a unit $e \in \widehat{\mathbf{Z H}}$ with $\Delta_{1}\left(A_{L}\right)=e\left(\mathscr{M}_{12} /\left(t_{1}-1\right)\right)$. If $\varepsilon: \overline{\mathbf{Z H}} \rightarrow \mathbf{Z}$ is the unique continuous extension of the locally constant homomorphism $\varepsilon: \mathbf{Z} H \rightarrow \mathbf{Z}$, then $\varepsilon(e)= \pm 1$, since $e$ is a unit; multiplying $\Delta_{1}\left(A_{L}\right)$ by -1 if necessary, we may as well assume that $\varepsilon(e)=1$.

For $r, s \geqslant 0$ let $\gamma(r, s)$ be the coefficient of $\left(t_{1}-1\right)^{r}\left(t_{2}-1\right)^{s}$ in $\mathscr{M}_{12} /\left(t_{1}-1\right)$, and let $\delta(r, s)$ be the g.c.d. of those $\gamma(u, v)$ with $u \leqslant r, v \leqslant s$, and $u+v<r+s$. Then since $\varepsilon(e)=1$,

$$
\frac{1}{r ! s !} \varepsilon\left(\frac{d^{r+s}}{d t_{1}^{r} d t_{2}^{s}}\left(\Delta_{1}\left(A_{L}\right)\right)\right) \equiv \gamma(r, s)
$$

(modulo $\delta(r, s))$.

To complete the proof, then, it suffices to show that for all $r, s \geqslant 0, \delta(r, s)=$ $\Delta([r+1, s+1])$ and $\gamma(r, s) \equiv(-1)^{s} \mu([r+1, s+1])$ (modulo $\left.\delta(r, s)\right)$. Note that $\delta(0,0)=0=\Delta([1,1])$, and $\gamma(0,0)=\mu(2,1)=(-1)^{0} \mu([1,1])$ is the linking number of the components of $L$.

Proceeding by induction on $r+s$, suppose $r+s \geqslant 1$. The inductive hypothesis directly implies that $\delta(r, s)=\Delta([r+1, s+1])$.

If $r \geqslant 1$, then

$$
\gamma(r, s)=\sum_{C(r, s)} \mu\left(c_{1}, \ldots, c_{r+s}, 2,1\right),
$$

and so, by Corollary (6.3) and cyclic symmetry [14, Theorem 6],

$$
\begin{gathered}
\gamma(r, s) \equiv(-1)^{s} \mu\left(1_{1}, \ldots, 1_{r+1}, 2_{1}, \ldots, 2_{s+1}\right) \\
\left(\operatorname{modulo} \Delta\left(1_{1}, \ldots, 1_{r+1}, 2_{1}, \ldots, 2_{s+1}\right)=\Delta\left(1_{1}, \ldots, 1_{r}, 2_{1}, \ldots, 2_{s+1}, 1\right)\right) .
\end{gathered}
$$


If $r=0$, on the other hand, then $\gamma(r, s)=\mu\left(2_{1}, \ldots, 2_{s+1}, 1\right)$. Noting that $\Delta\left(2_{1}, \ldots, 2_{s+1}, 1\right)=\Delta^{*}\left(2_{1}, \ldots, 2_{s+1}, 1\right)$, we may apply $[14$, assertion (27)] to conclude that

$\gamma(r, s) \equiv(-1)^{s+2} \mu([1, s+1]) \quad\left(\operatorname{modulo} \Delta\left(2_{1}, \ldots, 2_{s+1}, 1\right)=\Delta([1, s+1])\right)$.

Q.E.D.

That the congruence of Theorem (6.4) holds modulo $\Delta^{*}([r+1, s+1])$ was shown by K. Murasugi [15, Theorem 4.1]. Though the improvement seems modest, it leads to a considerable simplification of several other results due to Murasugi; compare [16, Lemma 7.3] with Corollary (6.5) below, for instance, or [16, Theorem 8.1] with the description of the Chen groups of $L$ in the next section. By the way, [15, Theorems 4.2 and 4.3] can also be deduced from our Theorem (4.1); we leave the details to the reader.

Recall that for $t \geqslant 2,(I H)^{t}$ consists of those elements of $I H$ all of whose partial derivatives of orders $<t$ are mapped to 0 by $\varepsilon$. From Theorem (6.4) we directly conclude

CoRollary (6.5). For $t \geqslant 0, \Delta_{1}\left(A_{L}\right) \in(I H)^{t}$ iff $\bar{\mu}([r+1, s+1])=0$ for all $r, s \geqslant 0$ with $r+s<t$. Consequently, $\Delta_{1}\left(A_{L}\right)=0$ iff $\bar{\mu}([r+1, s+1])=0$ for all $r, s \geqslant 0$.

Note that by Proposition (6.1), the greatest integer $t$ with $\Delta_{1}\left(A_{L}\right) \in(I H)^{t}$, if it exists, must be even.

Another interesting consequence of Theorem (6.4) is

COROLlary (6.6). Whenever $r+s$ is odd, $2 \bar{\mu}([r+1, s+1])=0$.

Proof. Let $L^{\prime}$ be the link obtained from $L$ by reversing the orientations of both of its components. By Torres' first relation [20], $\Delta_{1}\left(A_{L}\right)$ is also an Alexander polynomial for $L^{\prime}$. By Theorem (6.4), the $\bar{\mu}$-invariants $\bar{\mu}^{\prime}([r+1, s+1])$ associated to $L^{\prime}$ are related to those of $L$ : there is a fixed $\delta \in\{0,1\}$ such that $\bar{\mu}^{\prime}([r+1, s+1])=$ $(-1)^{\delta} \bar{\mu}([r+1, s+1]) \forall r, s \geqslant 0$. Also, according to J. Milnor [14, p. 296], $\bar{\mu}^{\prime}([r+1, s+1])=(-1)^{r+s} \bar{\mu}([r+1, s+1]) \forall r, s \geqslant 0$.

If $\bar{\mu}([r+1, s+1])=0 \forall r, s \geqslant 0$, then, of course, the conclusion holds.

If not, then there is a nonzero $\bar{\mu}\left(\left[r_{0}+1, s_{0}+1\right]\right)$ with $t_{0}=r_{0}+s_{0}$ as small as possible; then $\Delta_{1}\left(A_{L}\right) \in(I H)^{t_{0}}-(I H)^{t_{0}+1}$. Since $t_{0}$ must be even,

$$
\bar{\mu}^{\prime}\left(\left[r_{0}+1, s_{0}+1\right]\right)=(-1)^{t_{0}} \bar{\mu}\left(\left[r_{0}+1, s_{0}+1\right]\right)=\bar{\mu}\left(\left[r_{0}+1, s_{0}+1\right]\right) .
$$

Since $\Delta\left(\left[r_{0}+1, s_{0}+1\right]\right)=0$ by the minimality of $t_{0}$, and $\bar{\mu}^{\prime}\left(\left[r_{0}+1, s_{0}+1\right]\right)=$ $(-1)^{\delta} \bar{\mu}\left(\left[r_{0}+1, s_{0}+1\right]\right)$, necessarily $\delta=0$. Thus $(-1)^{r+s} \bar{\mu}([r+1, s+1])=$ $\bar{\mu}^{\prime}([r+1, s+1])=\bar{\mu}([r+1, s+1]) \forall r, s \geqslant 0$. Q.E.D.

J. H. Conway [3, p. 340] has observed that the Alexander polynomial of a + amphicheiral two-component link (i.e., one which is ambient isotopic to its mirror image) is 0. (Conway uses "amphicheiral" in this sense; see [3, p. 336].) Using Corollary (6.6), we generalize this somewhat: 
COROllaRy (6.7). If the $\bar{\mu}$-invariants of $L$ and its mirror image coincide, then $\Delta_{1}\left(A_{L}\right)=0$.

Proof. According to J. Milnor [14, p. 296], the $\bar{\mu}$-invariants $\bar{\mu}^{m}\left(i_{1}, \ldots, i_{q}\right)$ of the mirror image of $L$ are related to those of $L$ by $\bar{\mu}^{m}\left(i_{1}, \ldots, i_{q}\right)=(-1)^{q-1} \bar{\mu}\left(i_{1}, \ldots, i_{q}\right)$. By hypothesis, then,

$$
\bar{\mu}([r+1, s+1])=(-1)^{r+s+1} \bar{\mu}([r+1, s+1]) \quad \forall r, s \geqslant 0,
$$

so that $2 \bar{\mu}([r+1, s+1])=0$ whenever $r+s$ is even. Combining this with Corollary (6.6), we conclude immediately that there can be no nonzero $\bar{\mu}([r+1, s+1])$ with $r+s$ minimal, so $\bar{\mu}([r+1, s+1])=0 \forall r, s \geqslant 0$. By Theorem (6.4), then, $\Delta_{1}\left(A_{L}\right)$ $=0$. Q.E.D.

Corollary (6.7) does not generalize to the case $\mu=3$, since the Borromean rings $\left(6_{2}^{3}\right.$ of [18, Appendix C]) are + amphicheiral and have nonzero Alexander polynomial. On the other hand, any link of two or more components whose $\bar{\mu}$-invariants are the same as those of the link obtained by reversing the orientations of all the components of its mirror image (e.g., any - amphicheiral link) must have all its $\bar{\mu}$-invariants 0 (this follows from remarks of $[14$, p. 296]), and so must have $E_{\mu-1}\left(A_{L}\right)=0$, by Theorem (4.1).

7. The Chen groups. In the last two sections of the paper we revert to the general case $\mu \geqslant 2$, except where stated otherwise.

The associated graded module [1, III; $24, \mathrm{VIII}]$ of a finitely generated $\mathbf{Z} H$-module $B$ is the graded abelian group

$$
\operatorname{gr}(B)=\bigoplus_{q \geqslant 0}(I H)^{q} \cdot B /(I H)^{q+1} \cdot B
$$

it is a graded module over the graded $\operatorname{ring} \operatorname{gr}(\mathbf{Z} H)$ in a natural way. The associated graded module of the $I H$-adic completion $\hat{B}$ is defined analogously:

$$
\operatorname{gr}(\hat{B})=\bigoplus_{q \geqslant 0}(\widehat{I H})^{q} \cdot \hat{B} /(\widehat{I H})^{q+1} \cdot \hat{B}
$$

The natural mapping $h: \mathbf{Z} H \rightarrow \widehat{\mathbf{Z H}}$ induces an isomorphism $\operatorname{gr}(h): \operatorname{gr}(\mathbf{Z} H) \rightarrow$ $\operatorname{gr}(\widehat{\mathbf{Z H}})$ of graded rings, with respect to which $\operatorname{gr}(h): \operatorname{gr}(B) \rightarrow \operatorname{gr}(\hat{B})$ is an isomorphism of graded modules [1, III, §2.12].

Our interest in associated graded modules stems from the fact that for $q \geqslant 1$ the $(q+1)$ st Chen group of $L, G^{\prime \prime} G_{q+1} / G^{\prime \prime} G_{q+2}$, is isomorphic to $(I H)^{q-1} \cdot B_{L} /(I H)^{q}$ $\cdot B_{L}=\mathrm{gr}_{q-1}\left(B_{L}\right)$, and hence to $\mathrm{gr}_{q-1}\left(\hat{B}_{L}\right)[12]$.

Following [24, VIII, §1] in substance if not notation, for a finitely generated $L H$-modult $B$ we define the initial form function in: $\hat{B} \rightarrow \operatorname{gr}(\hat{B})$ as follows: if $x \in$ $(\widehat{I H})^{q} \cdot \hat{B}-(\widehat{I H})^{q+1} \cdot \hat{B}$ then $\operatorname{in}(x)=x+(\widehat{I H})^{q+1} \cdot \hat{B} \in \operatorname{gr}_{q}(\hat{B})$, and if $x \in$ $\bigcap_{q \geqslant 0}(\widehat{I H})^{q} \cdot \hat{B}$ then in $(x)=0$. If $K \subseteq \hat{B}$ is a $\widehat{\mathbf{Z H}}$-submodule, the leading submodule of $K$ is the $\operatorname{gr}(\widehat{\mathbf{Z H}})$-submodule of $\operatorname{gr}(\hat{B})$ generated by in $(K)$. If $g: \hat{B} \rightarrow C$ is a $\widehat{\mathbf{Z H}}$-epimorphism with kernel $K$, then $\operatorname{gr}(g): \operatorname{gr}(\hat{B}) \rightarrow \operatorname{gr}(C)$ is a $\operatorname{gr}(\widehat{\mathbf{Z H}})$-epimorphism whose kernel is the leading submodule of $K$. 
It follows from Theorem (3.5), then, that the $\operatorname{gr}(\widehat{\mathbf{Z H}})$-module $\operatorname{gr}\left(\hat{B}_{L}\right)$ is isomorphic to the quotient of $\operatorname{gr}\left(\hat{Z}_{2}\right)$ by the leading submodule of the image of $\left\langle\nu_{r}, \hat{\zeta}_{3}\right\rangle$, for every $r \in\{0, \ldots, \mu\}$. Unfortunately, the initial forms of the elements of a generating set of a submodule of a $\widehat{\mathbf{Z H}}$-module do not necessarily generate its leading submodule (see, for instance, [12, Example 2]), so we do not obtain a presentation of $\operatorname{gr}\left(\hat{B}_{L}\right)$ as a $\operatorname{gr}(\widehat{\mathbf{Z H}})$-module directly from Theorem (3.5). Nevertheless, we can prove the following theorem, due (for the most part) to J. A. Hillman [9, V].

THEOREM (7.1). Let $q \geqslant 1$. Then any two of these are equivalent:

(a) $E_{\mu-1}\left(A_{L}\right) \subseteq(I H)^{q}$,

(b) $E_{\mu-2}\left(B_{L}\right) \subseteq(I H)^{q-1+\left(\mu_{2}^{-1}\right)}$, and

(c) $G^{\prime \prime} G_{p} / G^{\prime \prime} G_{p+1} \cong \Phi^{\prime \prime} \Phi_{p} / \Phi^{\prime \prime} \Phi_{p+1} \forall p \in\{1, \ldots, q\}$.

Proof. Since (a), (b) and (c) are all true for $q=1$, we may restrict our attention to values $q \geqslant 2$.

According to [21, Theorem 1.1], $E_{\mu-1}\left(A_{L}\right) \cdot(I H)^{\left(\mu_{2}^{-1}\right)-1} \subseteq E_{\mu-2}\left(B_{L}\right)$ if $\mu \geqslant 3$, and $E_{\mu-1}\left(A_{L}\right)=E_{\mu-2}\left(B_{L}\right) \cdot I H$ if $\mu=2$. The implication (b) $\Rightarrow$ (a) follows immediately.

Note that if $L_{0}$ is the trivial link of $\mu$ components then its group is $\Phi$, and Theorem (3.5) asserts that $\hat{B}_{L_{0}} \cong \operatorname{coker} \hat{\zeta}_{3}$. Thus for $p \geqslant 2$ the $p$ th Chen group of $L_{0}$, $\Phi^{\prime \prime} \Phi_{p} / \Phi^{\prime \prime} \Phi_{p+1}$, is isomorphic to $\operatorname{gr}_{p-2}\left(\operatorname{coker} \hat{\zeta}_{3}\right)$. The condition (c), then, may be restated as $\operatorname{gr}_{p-2}\left(\hat{B}_{L}\right) \cong \operatorname{gr}_{p-2}\left(\operatorname{coker} \hat{\zeta}_{3}\right) \forall p \in\{2, \ldots, q\}$.

Suppose, now, that (a) holds. By Corollary (4.3), it follows that $(I H)^{q}$ also contains $E_{\mu-1}\left(\mathscr{M}_{q}\right)$, the ideal of $\mathbf{Z} H$ generated by the entries of $\mathscr{M}_{q}$. Equivalently, every entry of $\mathscr{M}$ is an element of $(\widehat{I H})^{q}$, and so every entry of $\mathscr{N}$ is an element of $(\widehat{I H})^{q-1}$. Then $\operatorname{ker} \tau+(\widehat{I H})^{q-1} \cdot \hat{Z}_{2}=\hat{\zeta}_{3}\left(\hat{Z}_{3}\right)+(\widehat{I H})^{q-1} \cdot \hat{Z}_{2}$, so $\tau$ induces an isomorphism between coker $\hat{\zeta}_{3} /(\widehat{I H})^{q-1} \cdot \operatorname{coker} \hat{\zeta}_{3}$ and $\hat{B}_{L} /(\widehat{I H})^{q-1} \cdot \hat{B}_{L}$; certainly then $\operatorname{gr}_{p-2}\left(\operatorname{coker} \hat{\zeta}_{3}\right) \cong \operatorname{gr}_{p-2}\left(\hat{B}_{L}\right)$ for $2 \leqslant p \leqslant q$. This verifies the implication (a) $\Rightarrow$ (c).

Finally, suppose (c) holds. Since $\hat{\zeta}_{3}\left(\hat{Z}_{3}\right) \subseteq \operatorname{ker} \tau$, there is a $\widehat{\mathbf{Z H}}$-epimorphism

$$
\bar{\tau} \text { : coker } \hat{\zeta}_{3} /(\widehat{I H})^{q-1} \cdot \operatorname{coker} \hat{\zeta}_{3} \rightarrow \hat{B}_{L} /(\widehat{I H})^{q-1} \cdot \hat{B}_{L}
$$

induced by $\tau$. The $\widehat{I H}$-adic topology on $\hat{B}_{L} /(\widehat{I H})^{q-1} \cdot \hat{B}_{L}$ is obviously discrete, so by $[1$, III, §2.8, Theorem 1] we may conclude that

$$
\operatorname{gr}_{p-2}(\bar{\tau}): \operatorname{gr}_{p-2}\left(\operatorname{coker} \hat{\zeta}_{3} /(\widehat{I H})^{q-1} \cdot \operatorname{coker} \hat{\zeta}_{3}\right) \rightarrow \operatorname{gr}_{p-2}\left(\hat{B}_{L} /(\widehat{I H})^{q-1} \cdot \hat{B}_{L}\right)
$$

is surjective for every integer $p \geqslant 2$. For $p>q, \operatorname{gr}_{p-2}\left(\operatorname{coker} \hat{\zeta}_{3} /(\widehat{I H})^{q-1} \cdot \operatorname{coker} \hat{\zeta}_{3}\right)$ $=0$, so $\operatorname{gr}_{p-2}(\bar{\tau})$ is, in fact, an isomorphism. Furthermore, for $2 \leqslant p \leqslant q$,

$$
\operatorname{gr}_{p-2}\left(\operatorname{coker} \hat{\zeta}_{3} /(\widehat{I H})^{q-1} \cdot \operatorname{coker} \hat{\zeta}_{3}\right)=\operatorname{gr}_{p-2}\left(\operatorname{coker} \hat{\zeta}_{3}\right)
$$

and

$$
\operatorname{gr}_{p-2}\left(\hat{B}_{L} /(\widehat{I H})^{q-1} \cdot \hat{B}_{L}\right)=\operatorname{gr}_{p-2}\left(\hat{B}_{L}\right)
$$

are isomorphic finitely generated abelian groups, by (c), so by the Hopfian property of such groups the epimorphism $\operatorname{gr}_{p-2}(\bar{\tau})$ must be an isomorphism. Thus $\operatorname{gr}_{p-2}(\bar{\tau})$ is an isomorphism for every $p \geqslant 2$, so $\bar{\tau}$ itself must be an isomorphism, by [1, III, $\S 2.8$, Corollary 3]. 
A presentation matrix for the $\widehat{\mathbf{Z H}}$-module $\hat{B}_{L} /(\widehat{I H})^{q-1} \cdot \hat{B}_{L}$ can be obtained from any presentation matrix for $\hat{B}_{L}$ by adjoining a number of rows whose entries are all in $(\widehat{I H})^{q-1}$; by [22, Lemma 3.1], then,

$$
\sum_{i \geqslant 0} E_{\mu-2+i}\left(\hat{B}_{L}\right) \cdot(\widehat{I H})^{(q-1) i}=\sum_{i \geqslant 0} E_{\mu-2+i}\left(\hat{B}_{L} /(\widehat{I H})^{q-1} \cdot \hat{B}_{L}\right) \cdot(\widehat{I H})^{(q-1) i} .
$$

Similarly, since $N_{3}(\mu)$ is a presentation matrix for coker $\hat{\zeta}_{3}$,

$$
\begin{aligned}
& \sum_{i \geqslant 0} E_{\mu-2+i}\left(N_{3}(\mu)\right) \cdot(\widehat{I H})^{(q-1) i} \\
& \quad=\sum_{i \geqslant 0} E_{\mu-2+i}\left(\operatorname{coker} \hat{\zeta}_{3} /(\widehat{I H})^{q-1} \cdot \operatorname{coker} \hat{\zeta}_{3}\right) \cdot(\widehat{I H})^{(q-1) i} .
\end{aligned}
$$

Since $\hat{B}_{L} /(\widehat{I H})^{q-1} \cdot \hat{B}_{L}$ and coker $\hat{\zeta}_{3} /(\widehat{I H})^{q-1} \cdot \operatorname{coker} \hat{\zeta}_{3}$ are isomorphic $\widehat{\mathbf{Z H}}$-modules, they have the same elementary ideals, and hence

$$
\sum_{i \geqslant 0} E_{\mu-2+i}\left(\hat{B}_{L}\right) \cdot(\widehat{I H})^{(q-1) i}=\sum_{i \geqslant 0} E_{\mu-2+i}\left(N_{3}(\mu)\right) \cdot(\widehat{I H})^{(q-1) i} .
$$

For $1 \leqslant i \leqslant\left(\begin{array}{c}\mu \\ 2\end{array}\right)-\mu+2=\left(\begin{array}{c}\mu-1 \\ 2\end{array}\right)+1, E_{\mu-2+i}\left(N_{3}(\mu)\right)$, the ideal of $\widehat{\mathbf{Z H}}$ generated by the determinants of the $\left(\left({ }_{2}^{\mu-1}\right)+1-i\right) \times\left(\left({ }_{2}^{\mu-1}\right)+1-i\right)$ submatrices of $N_{3}(\mu)$, is certainly contained in $\left(\widehat{I H}^{\left(\mu_{2}^{-1}\right)+1-i}\right.$, since every entry of $N_{3}(\mu)$ is an element of $\widehat{I H}$. Furthermore, $E_{\mu-2}\left(N_{3}(\mu)\right)=0[6,(5.1)]$, and hence

$$
\sum_{i \geqslant 0} E_{\mu-2+i}\left(N_{3}(\mu)\right) \cdot(\widehat{I H})^{(q-1) i} \subseteq(\widehat{I H})^{\left(\mu_{2}-1\right)+q-1} .
$$

Thus $E_{\mu-2}\left(\hat{B}_{L}\right) \subseteq(\widehat{I H})^{\left(\mu_{2}^{-1}\right)+q-1}$, so since $E_{\mu-2}\left(\hat{B}_{L}\right)$ is the ideal of $\widehat{\mathbf{Z H}}$ generated by $E_{\mu-2}\left(B_{L}\right)$, necessarily $(I H)^{\left(\mu_{2}-1\right)+q-1} \supseteq E_{\mu-2}\left(B_{L}\right)$.

The proof of the implication (c) $\Rightarrow$ (b) completes the proof of the theorem. Q.E.D.

In the particular case $\mu=2$, the image of $\left\langle\nu_{1}, \hat{\zeta}_{3}\right\rangle=\nu_{1}$ is a principal ideal in $\hat{\mathbf{Z}}_{2}=\widehat{\mathbf{Z H}}$. It is not difficult to deduce that the leading submodule of the image of $\nu_{1}$ is generated by the initial form of any generator of this ideal, so that in this case Theorem (3.5) does yield an explicit description of the Chen groups of $L$, closely related to that found by K. Murasugi [16, Theorem 8.1], as follows.

If $L \subseteq S^{3}$ is a tame link of $\mu=2$ components, let $v$ be the greatest integer with $\Delta_{1}\left(A_{L}\right) \in(I H)^{v}$, if any such integer exists, and $v=\infty$ otherwise. Equivalently, $v$ is the least integer with $\mu([r+1, s+1]) \neq 0$ for some $r, s \geqslant 0$ with $r+s=v$, if any such integer exists. If $v$ is finite, let $d>0$ be the g.c.d. of the $\mu([r+1, s+1])$ with $r+s=v$; we denote by $\mathbf{Z}_{d}$ the cyclic group of order $d$. Then we have

THEOREM (7.2). If $v=\infty, G^{\prime \prime} G_{p} / G^{\prime \prime} G_{p+1} \cong \mathbf{Z}^{p-1} \forall p \geqslant 2$.

If $v$ is finite, then $G^{\prime \prime} G_{p} / G^{\prime \prime} G_{p+1} \cong \mathbf{Z}^{p-1} \forall p \in\{2, \ldots, v+1\}$, and $G^{\prime \prime} G_{p} / G^{\prime \prime} G_{p+1}$ $\cong \mathbf{Z}^{v} \oplus \mathbf{Z}_{d}^{p^{-v-1}} \forall p \geqslant v+2$.

Proof. If $v=\infty$, then $\mathscr{M}_{12}=0$ (see the proof of Theorem (6.4)), so $\mathscr{N}_{12}=0$, so by Theorem (3.5) $\hat{B}_{L} \cong \widehat{\mathbf{Z H}}$. Hence for $p \geqslant 2$,

$$
G^{\prime \prime} G_{p} / G^{\prime \prime} G_{p+1} \cong \operatorname{gr}_{p-2}(\widehat{\mathbf{Z H}})=(\widehat{I H})^{p-2} /(\widehat{I H})^{p-1}
$$

is isomorphic to $\mathbf{Z}^{p-1}$. 
If $v$ is finite, on the other hand, then

$$
\operatorname{in}\left(\mathscr{N}_{12}\right)=\sum_{s=0}^{v}(-1)^{s} \mu([v-s+1, s+1])\left(t_{1}-1\right)^{v-s}\left(t_{2}-1\right)^{s}+(I H)^{v+1}
$$

(again, see the proof of Theorem (6.4)). That $\operatorname{gr}\left(\hat{B}_{L}\right)$ is isomorphic to the quotient of $\operatorname{gr}(\widehat{\mathbf{Z H}})$ by the graded ideal generated by $\operatorname{in}\left(\mathcal{N}_{12}\right)$ implies that, for $p \leqslant v+1$, $G^{\prime \prime} G_{p} / G^{\prime \prime} G_{p+1} \cong \operatorname{gr}_{p-2}\left(\hat{B}_{L}\right)$ is isomorphic to $\operatorname{gr}_{p-2}(\overline{\mathbf{Z H}}) \cong \mathbf{Z}^{p-1}$. For $p \geqslant v+2$, on the other hand, $\operatorname{gr}_{p-2}(\tau): \mathrm{gr}_{p-2}(\widehat{\mathbf{Z H}}) \rightarrow \operatorname{gr}_{p-2}\left(\hat{B}_{L}\right)$ is an epimorphism whose kernel is the subgroup of $\operatorname{gr}_{p-2}(\widehat{\mathrm{ZH}})=(\widehat{I H})^{p-2} /(\overline{I H})^{p-1}$ generated by the elements

$$
\sum_{s=0}^{v}(-1)^{s} \mu([v-s+1, s+1])\left(t_{1}-1\right)^{v-s+t}\left(t_{2}-1\right)^{s+p-2-v-t}+(\widehat{I H})^{p-1}
$$

$0 \leqslant t \leqslant p-2-v$. Thus $G^{\prime \prime} G_{p} / G^{\prime \prime} G_{p+1} \cong \operatorname{gr}_{p-2}\left(\hat{B}_{L}\right)$ is a Z-module with a $(p-1$ $-v) \times(p-1)$ presentation matrix whose $i j$ entry is 0 if $j<i$ or $j>i+v$, and $(-1)^{j-i} \mu([v-j+i+1, j-i+1])$ if $i \leqslant j \leqslant i+v$. That $G^{\prime \prime} G_{p} / G^{\prime \prime} G_{p+1} \cong \mathbf{Z}^{v} \oplus$ $\mathbf{Z}_{d}^{p^{-v-1}}$ follows from the calculation of the elementary ideals of such a matrix, which can be accomplished with a relatively simple inductive argument [16, Lemma 8.1]. Q.E.D.

Alternatively, this theorem could be proven directly from [16, Theorem 8.1], using Theorem (6.4) to describe the integers $A^{(q)}(L)$ defined in [16] in terms of the various integers $\mu([r+1, s+1])$; since the proof of [16, Theorem 8.1] is rather involved, though, we have included the more self-contained argument just given.

Having found that the Alexander polynomial of a two-component link determines its Chen groups (as we have just seen), K. Murasugi $[16, \S 1]$ raised the possibility that the Chen groups of a $\mu$-component link, $\mu \geqslant 3$, may be determined by the Alexander polynomial of the link, together with those of its sublinks. This turns out not to be the case, as we proceed to show by example.

For $\mu \geqslant 4$, J. Milnor [13, Figure 7] has given an example of a $\mu$-component tame link $L$ in $S^{3}$ with these properties: its Alexander polynomial $\Delta_{1}\left(A_{L}\right)=0$, every one of its proper sublinks is trivial, and the only sequence $\left(i_{1}, \ldots, i_{p}, \mu-1, \mu\right)$ of pairwise distinct elements of $\{1, \ldots, \mu\}$ with $\mu\left(i_{1}, \ldots, i_{p}, \mu-1, \mu\right) \neq 0$ is $\left(i_{1}, \ldots, i_{p}, \mu\right.$ $-1, \mu)=(1, \ldots, \mu)$. The latter property clearly implies that the coefficient of $\left(t_{\mu}-1\right) \cdot \prod_{i=1}^{\mu-2}\left(t_{i}-1\right)$ in $\mathscr{M}_{\mu \mu-1}$ is not 0 , and consequently $\mathscr{M}_{\mu} \neq 0$. By Corollary (4.3), then, $E_{\mu-1}\left(A_{L}\right) \nsubseteq(I H)^{\mu}$, and so by Theorem (7.1) at least one of the first $\mu$ Chen groups of $L$ must not be isomorphic to the corresponding Chen group of the trivial $\mu$-component link, $L_{0}$. However, $L, L_{0}$, and all their sublinks of two or more components have Alexander polynomial 0 , while all their one-component sublinks have Alexander polynomial 1.

For $\mu=3$, the link of [13, Figure 7], the Borromean rings, has nonzero Alexander polynomial. However, if $L$ is the link obtained from the Borromean rings by doubling the first component ( $L$ is pictured in the lower left-hand corner of $[18, \mathrm{p}$. 138]), then $\Delta_{1}\left(A_{L}\right)=0$ and

$$
E_{2}\left(A_{L}\right)=\left(t_{1}-1\right)^{2}\left(t_{2}-1\right)\left(t_{3}-1\right) \cdot\left(t_{2}-1, t_{3}-1\right) .
$$


Thus $L$ and the trivial three-component link both have Alexander polynomial 0 , all the proper sublinks of either are trivial, and we conclude from Theorem (7.1) that their sixth Chen groups are not isomorphic.

8. Some questions. If $L \subseteq S^{3}$ is a tame link of $\mu$ components, then it follows from Corollary (4.3) that for $q \geqslant 2, E_{\mu-1}\left(A_{L}\right) \subseteq(I H)^{q}$ iff $\mathscr{M}_{q}=0$. We immediately conclude

Proposition (8.1). If $\mu\left(i_{1}, \ldots, i_{p}\right)=0$ whenever $2 \leqslant p \leqslant q$, then $E_{\mu-1}\left(A_{L}\right) \subseteq$ $(I H)^{q}$.

Since $\bigcap_{q}(I H)^{q}=0$, we also have

Corollary (8.2). If $\mu\left(i_{1}, \ldots, i_{p}\right)=0$ whenever $2 \leqslant p$, then $E_{\mu-1}\left(A_{L}\right)=0$.

To what extent do the converses of these two statements hold? If $q=2$, the converse of Proposition (8.1) follows immediately from the definition of $\mathscr{M}$; this is already known [22, Theorem 2]. At first glance, though, the prospects for $q \geqslant 3$ seem $\operatorname{dim}$, given the potential cancellation apparent in the definition of the entries of $\mathscr{M}$. However, the integers $\mu\left(i_{1}, \ldots, i_{q}\right)$ satisfy many relations (cyclic symmetry [14, Theorem 6] and the relations of [2, Lemma (3.3)], for instance), and using these we have been able to verify the converse of Proposition (8.1) for $q=3,4$ and 5. We will not present these arguments here, though, since they are cumbersome and represent only an incomplete answer to our question.

For $q \geqslant 2, \mu\left(i_{1}, \ldots, i_{p}\right)=0$ whenever $2 \leqslant p \leqslant q$ iff $G / G_{r} \cong \Phi / \Phi_{r}$ for $3 \leqslant r \leqslant q$ +1 (this is a simple consequence of [2, Corollary (3.6)]). By Theorem (7.1), then, Proposition (8.1) is equivalent to the assertion that if $G / G_{r} \cong \Phi / \Phi_{r}$ for $3 \leqslant r \leqslant q+$ 1 , then $G^{\prime \prime} G_{p} / G^{\prime \prime} G_{p+1} \cong \Phi^{\prime \prime} \Phi_{p} / \Phi^{\prime \prime} \Phi_{p+1}$ for $2 \leqslant p \leqslant q$.

In the particular case $\mu=2$, Corollary (6.5) indicates that Proposition (8.1) is equivalent to the assertion that if $\mu\left(i_{1}, \ldots, i_{p}\right)=0$ whenever $2 \leqslant p \leqslant q$, then $\mu([r+1, s+1])=0$ whenever $0 \leqslant r+s \leqslant q-2$. In this case the converse can also be verified for $q=6$, using some relations from [14, §4].

An interesting consequence of the converse of Proposition (8.1) would be that a link with $E_{\mu-1}\left(A_{L}\right) \subseteq(I H)^{\mu}$ would have $\mu\left(i_{1}, \ldots, i_{p}\right)=0$ whenever $i_{1}, \ldots, i_{p}$ are pairwise distinct, and so would be homotopically trivial $[13, \S 5]$. For $2 \leqslant \mu \leqslant 5$ this consequence is true, because of our partial verification of the converse of Proposition (8.1). In addition, it is not difficult to show that, for $\mu=2$ or $3, E_{\mu-1}\left(A_{L}\right) \subseteq(I H)^{\mu}$ if and only if $L$ is homotopically trivial.

\section{BIBLIOGRAPHY}

1. N. Bourbaki, Commutative algebra, Hermann, Paris and Addison-Wesley, Reading, Mass., 1972.

2. K.-T. Chen, R. H. Fox and R. C. Lyndon, Free differential calculus. IV, Ann. of Math. (2) 68 (1958), $81-95$

3. J. H. Conway, An enumeration of knots and links, and some of their algebraic properties, Computational Problems in Abstract Algebra, Pergamon Press, Oxford, 1969, pp. 329-358.

4. R. H. Crowell, The derived module of a homomorphism, Adv. in Math. 6 (1971), 210-238.

5. ___ Torsion in link modules, J. Math. Mech. 14 (1965), 289-298.

6. R. H. Crowell and D. Strauss, On the elementary ideals of link modules, Trans. Amer. Math. Soc. 142 (1969), 93-109. 
7. R. H. Fox, Free differential calculus. I, Ann. of Math. (2) 57 (1953), 547-560.

8. __ Free differential calculus. II, Ann. of Math. (2) 59 (1954), 196-210.

9. J. A. Hillman, Alexander ideals of links, Springer-Verlag, Berlin and New York, 1981.

10. N. Jacobson, Basic algebra I, Freeman, San Francisco, Calif., 1974.

11. M. E. Kidwell, On the Alexander polynomials of certain three-component links, Proc. Amer. Math. Soc. 71 (1978), 351-354.

12. W. S. Massey, Completion of link modules, Duke Math. J. 47 (1980), 399-420.

13. J. Milnor, Link groups, Ann. of Math. (2) 59 (1954), 177-195.

14. Isotopy of links, Algebraic Geometry and Topology, Princeton Univ. Press, Princeton, N. J., 1957, pp. 280-306.

15. K. Murasugi, On Milnor's invariant for links, Trans. Amer. Math. Soc. 124 (1966), 94-110.

16. _ On Milnor's invariant for links. II. The Chen group, Trans. Amer. Math. Soc. 148 (1970), 41-61.

17. D. G. Northcott, Finite free resolutions, Cambridge Univ. Press, Cambridge, 1976.

18. D. Rolfsen, Knots and links, Publish or Perish, Berkeley, Calif., 1976.

19. N. Smythe, Isotopy invariants of links and the Alexander matrix, Amer. J. Math. 89 (1967), 693-704.

20. G. Torres, On the Alexander polynomial, Ann. of Math. (2) 57 (1953), 57-89.

21. L. Traldi, The determinantal ideals of link modules. I, Pacific J. Math. 101 (1982), 215-222.

22. , Linking numbers and the elementary ideals of links, Trans. Amer. Math. Soc. 275 (1983), 309-318.

23. Some properties of the determinantal ideals of link modules, Kobe J. Math. (to appear).

24. O. Zariski and P. Samuel, Commutative algebra, Vol. II, Van Nostrand, Princeton, N. J., 1960.

Department of Mathematics, Lafayette College, Easton, Pennsylvania 18042 